\title{
Impact of high-order tidal terms on binary neutron-star waveforms
}

\author{
Xisco Jiménez Forteza, ${ }^{1,2, *}$ Tiziano Abdelsalhin, ${ }^{2,3, \dagger}$ Paolo Pani, ${ }^{2,3, *}$ and Leonardo Gualtieri ${ }^{2,3, \S}$ \\ ${ }^{1}$ Sezione INFN Napoli, Complesso Universitario di Monte S. Angelo, Via Cinthia, I-80126, Napoli, Italy \\ ${ }^{2}$ Dipartimento di Fisica, “Sapienza” Università di Roma, Piazzale Aldo Moro 5, 00185, Roma, Italy \\ ${ }^{3}$ Sezione INFN Romal, Piazzale Aldo Moro 5, 00185, Roma, Italy
}

GW170817, the milestone gravitational-wave event originated from a binary neutron star merger, has allowed scientific community to place a constraint on the equation of state of neutron stars by extracting the leadingorder, tidal-deformability term from the gravitational waveform. Here we incorporate tidal corrections to the gravitational-wave phase at next-to-leading and next-to-next-to-leading order, including the magnetic tidal Love numbers, tail effects, and the spin-tidal couplings recently computed in Tiziano Abdelsalhin et al. [Phys. Rev. D 98, 104046 (2018)]. These effects have not yet been included in the waveform approximants for the analysis of GW170817. We provide a qualitative and quantitative analysis of the impact of these new terms by studying the parameter bias induced on events compatible with GW170817 assuming second-generation (advanced LIGO) and third-generation (Einstein Telescope) ground-based gravitational-wave interferometers. We find that including the tidal-tail term deteriorates the convergence properties of the post-Newtonian expansion in the relevant frequency range. We also find that the effect of magnetic tidal Love numbers could be measurable for an optimal GW170817 event with signal-to-noise ratio $\rho \approx 1750$ detected with the Einstein Telescope. On the same line, spin-tidal couplings may be relevant if mildly high-spin $\chi \gtrsim 0.1$ neutron star binaries exist in nature.

$$
\text { published journal version: }
$$

Phsyical Review D 98, 124014 (2018)

doi:10.1103/PhysRevD.98.124014

\section{INTRODUCTION}

In August 2017, the LIGO-Virgo Scientific Collaboration reported the milestone detection of gravitational waves (GWs) from a binary neutron star (BNS) coalescence [1], dubbed GW170817. This landmark discovery has opened a new era in astrophysics. Along with the GW detection, several telescopes also reported the observation of electromagnetic coincidence signals in various bands, inaugurating the birth of the GW multimessenger astronomy.

One of the most relevant implications of this discovery is arguably the possibility of constraining the equation of state (EoS) of the neutron-star (NS) core through the measurement of the tidal deformability of the binary components [18]. GW170817 allowed one to place stringent constraints on the leading-order, tidal-deformability parameter, which measures the induced quadrupole moment of the binary components due to tidal forces during the late inspiral. Though in the past some constraints on the radius of isolated NSs (and hence on the EoS) have been set based on electromagnetic observations (see Ref. [9] for a review), the GW channel is expected to provide more robust and tighter constraints, especially as more events are observed by current and next-generation GW detectors.

GW searches and parameter estimation pipelines rely on waveform approximants that accurately describe the inspiral, merger, and ringdown phases of the coalescence. While the early inspiral is accurately described by the postNewtonian (PN) theory [10-12], this description breaks down near the merger. To overcome this limitation, current waveform templates are recalibrated by fitting the late inspiral

\footnotetext{
*fjimenez@na.infn.it

$\dagger$ tiziano.abdelsalhin@roma1.infn.it

\$ paolo.pani@roma1.infn.it

§leonardo.gualtieri@roma1.infn.it
}

and merger phase to numerical relativity (NR) solutions [1316], producing the so-called Phenom [17-20] and SEOBNR approximants [21-26], the latter based on the effectiveone-body (EOB) formalism [27-30]. The weak-field (lowfrequency) regime is well described as a combination of pointparticle PN dynamics [31] with extra finite-size tidal corrections encoded in the tidal Love numbers (TLNs) [32]. NRcalibrated phenomenological models are constrained to recover the low-frequency solutions while correcting the deviations of the higher-order coefficients, which become important in the high-frequency regime. Thus, any new term included in the PN equations would also propagate to full waveform approximants, possibly in a contrived and nonlinear way.

Tidal deformability terms in the GW phase appear at 5PN order, but are magnified by the fifth power of $G R /\left(c^{2} M\right)$, where $M$ and $R$ are the stellar mass and radius, respectively. Thus, less compact stars (which are also more deformable) have a larger impact in the waveform relative to the pointparticle phase.

For nonspinning objects, the TLNs are naturally separated into two classes: those related with induced mass multipole moments (the so-called electric TLNs), and those related to the induced current multipole moments (the so-called magnetic TLNs). When the object is spinning, angular momentum gives rise to spin-tidal coupling and to a new class of rotational TLNs (RTLNs) [33-37].

Up to now, TaylorF2 approximants [38-40], which are based on the PN expansion of the orbital equations, properly account only for the contribution of the electric TLNs at the leading (5PN) and next-to-leading (6PN) order. On the other hand, tidal terms in EOB models have been partially included up to 7.5PN order, where the tidal-tail terms up to 7.5PN order emerge naturally from the expansion of the full EOB dynamics [41]. EOB models including tidal effects have been recently improved by the resummed time-domain version [42], but neglecting the effect of the spin, the magnetic TLNs, and the electric TLNs higher than the quadrupole [41].

The scope of this work is to quantify the effects of the 
higher-order tidal terms, namely the magnetic TLNs [4345] (whose leading contribution enters at 6PN order), of the tidal-tail terms [41] (whose leading-order contribution enters at $6.5 \mathrm{PN}$ order), and of the recently computed spin-tidal terms $[46,47]$ (which enter at $6.5 \mathrm{PN}$ order and are linear in the binary-component spins). Although these effects are presumably small, they might be important for several reasons: (i) Neglecting them might introduce systematics in the parameter estimation. This is especially important for the estimate of the tidal deformability, whose relative signal-to-noise ratio (SNR) accumulates mostly at high frequency, where the PN expansion is poorly convergent. (ii) Higher-order tidal terms could be used together with simulations to recalibrate effective models, thus obtaining a more precise approximation of the GW signal at high frequency. (iii) Spin-tidal couplings may break some of the degeneracy between tidal and spin effects, thus improving the parameter estimation of both quantities.

\section{TIDAL DEFORMATIONS OF NEUTRON STARS IN COALESCING BINARY SYSTEMS}

The theory of tidal deformation of compact bodies in general relativity has been developed in [43, 48-50], for nonrotating bodies, and then extended to rotating bodies in [33, 35, 51-53]. This theory has then been applied to compact binary systems, in order to compute the contribution of the tidal deformation to the emitted gravitational waveform, in $[41,54,55]$ for nonrotating NSs, and in [46, 47] for rotating stars.

\section{A. Tidal Love numbers of a spinning neutron star}

When a static, isolated and spherically symmetric object is perturbed by an external tidal field, its mass and current multipole moments [56-58] (see also [59]) are deformed. In the adiabatic approximation, in which the external tidal field is (adiabatically) static over the timescale of the star's response, the mass and current multipole moments (within first-order perturbation theory) are proportional to the electric and magnetic components of the tidal field. If the NS is not rotating ${ }^{1}$

$$
Q^{L}=\lambda_{l} G^{L} ; \quad S^{L}=\frac{\sigma_{l}}{c^{2}} H^{L}
$$

where $Q^{L}$ and $S^{L}$ are mass and current multipole moments of order $l$, respectively; $G^{L}$ and $H^{L}$ are electric and magnetic tidal tensors of order $l$ evaluated on the star's location; and $\lambda_{l}$ and $\sigma_{l}$ are electric and magnetic TLNs (we follow the notations and conventions of [46]). We remark that in the nonspinning case an $l$-pole tidal field can only induce an $l$-pole moment with the same parity. If the object rotates, instead, moments with different orders $l$ and $l^{\prime}=l \pm 1$ and with opposite parity are coupled to linear order in the spin. For $l=2,3$, Eqs. (1)

\footnotetext{
${ }^{1}$ We use the symmetric-trace-free (STF) notation [58] where capital letters in the middle of the alphabet $L, K$, etc. are shorthand for multi-indices $a_{1} \ldots a_{l}, b_{1} \ldots b_{k}$, etc., and round ( ), square [ ], and angular $\langle>$ brackets in the indices indicate symmetrization, antisymmetrization and trace-free symmetrization, respectively.
}

generalize to

$$
\begin{aligned}
Q^{a b} & =\lambda_{2} G^{a b}+\frac{\lambda_{23}}{c^{2}} J^{c} H^{a b c} \\
Q^{a b c} & =\lambda_{3} G^{a b c}+\frac{\lambda_{32}}{c^{2}} J^{\langle c} H^{a b\rangle} \\
S^{a b} & =\frac{\sigma_{2}}{c^{2}} H^{a b}+\sigma_{23} J^{c} G^{a b c} \\
S^{a b c} & =\frac{\sigma_{3}}{c^{2}} H^{a b c}+\sigma_{32} J^{\langle c} G^{a b\rangle}
\end{aligned}
$$

where we have neglected the multipole moments and the tidal tensors with $l>3$. In Eq. (2), $J^{a}$ is the spin vector of the star, and $\lambda_{l l^{\prime}}$ and $\sigma_{l l^{\prime}}$ are the RTLNs [33-35]. For an $N$-body system, the TNLs and RTLNs are denoted $\lambda_{l}^{(A)}, \sigma_{l}^{(A)}, \lambda_{l l^{\prime}}^{(A)}, \sigma_{l l^{\prime}}^{(A)}$, where $A=1,2, \ldots$ refers to the $A$ th body of the system.

The TLNs (and the RTLNs), computed by employing the relativistic perturbation theory of compact stars, depend on the NS EoS [33-35, 37, 43, 49, 50]; indeed, a stiffer EoS corresponds to a more deformable NS, and thus to higher TLNs, whereas a softer EoS corresponds to lower TLNs and RTLNs.

\section{B. Gravitational waveform of tidally deformed compact binaries up to 6.5PN order}

The largest tidal deformation of NSs occurs in the last stages of a compact binary coalescence. In this process, the binary system emits a strong GW signal, which depends on the TLNs and on the RTLNs of the NSs that are coalescing.

The signal emitted during the inspiral can be described by the PN formalism, recalibrated by fitting unknown (and possibly resummed) higher-order coefficients to NR solutions. The leading-order contributions of tidal deformation to the waveform appear at 5PN order; they do not depend on the NS spin and have been computed in [48]. The next-to-leading order contributions, which also do not depend on the spin and appear at 6PN order, have been computed in $[44,54,55]$. The (nonspinning) tail component (which appears at 6.5PN order) has been computed in [41]. Finally, the complete 6.5PN tidal waveform, which depends linearly on the NS spin, has recently been computed in [46]. For the sake of clarity, we show here the explicit expression for the tidal contribution to the waveform, up to 6.5PN order,

$$
\begin{aligned}
\psi_{T}(x) & =\frac{3}{128 v x^{5 / 2}}\left\{-\frac{39}{2} \tilde{\Lambda} x^{5}\right. \\
& \left.+(\delta \Lambda+\tilde{\Sigma}) x^{6}+(\hat{\Lambda}+\hat{\Sigma}+\hat{\Gamma}+\hat{K}) x^{6.5}+O\left(x^{7}\right)\right\},
\end{aligned}
$$

where ${ }^{2}$

$$
\begin{aligned}
\tilde{\Lambda} & =\frac{16}{13}\left(\frac{12}{\eta_{1}}-11\right) \eta_{1}^{5} \Lambda_{1}+(1 \leftrightarrow 2), \\
\delta \Lambda & =\left(\frac{5095}{28}-\frac{15895}{28 \eta_{1}}+\frac{5715 \eta_{1}}{14}-\frac{325 \eta_{1}^{2}}{7}\right) \eta_{1}^{5} \Lambda_{1} \\
& +(1 \leftrightarrow 2), \\
\tilde{\Sigma} & =\left(\frac{6920}{7}-\frac{20740}{21 \eta_{1}}\right) \eta_{1}^{5} \Sigma_{1}+(1 \leftrightarrow 2),
\end{aligned}
$$

\footnotetext{
2 Note that we slightly changed the notation with respect to [46], to be consistent with the notation of the LIGO-Virgo papers.
} 


$$
\begin{aligned}
\hat{\Lambda} & =\left[\left(\frac{593}{4}-\frac{1105}{8 \eta_{1}}+\frac{567 \eta_{1}}{8}-81 \eta_{1}^{2}\right) \chi_{2}\right. \\
& \left.+\left(-\frac{6607}{8}+\frac{6639 \eta_{1}}{8}-81 \eta_{1}^{2}\right) \chi_{1}\right] \eta_{1}^{5} \Lambda_{1}+(1 \leftrightarrow 2), \\
\hat{\Sigma} & =\left[\left(-\frac{9865}{3}+\frac{4933}{3 \eta_{1}}+1644 \eta_{1}\right) \chi_{2}-\chi_{1}\right] \eta_{1}^{5} \Sigma_{1} \\
& +(1 \leftrightarrow 2), \\
\hat{K} & =\frac{39}{2} \pi \tilde{\Lambda}, \\
\hat{\Gamma} & =\frac{\chi_{1}}{M^{4}}\left[\left(856 \eta_{1}-816 \eta_{1}^{2}\right) \lambda_{23}^{(1)}-\left(\frac{833 \eta_{1}}{3}-278 \eta_{1}^{2}\right) \sigma_{23}^{(1)}\right. \\
& \left.-v\left(272 \lambda_{32}^{(1)}-204 \sigma_{32}^{(1)}\right)\right]+(1 \leftrightarrow 2),
\end{aligned}
$$

where $\eta_{A}=M_{A} / M, M=M_{1}+M_{2}$ is the total mass of the binary, $M_{A}$ is the (Newtonian) mass of the Ath body, $v=\eta_{1} \eta_{2}$ is the symmetric mass ratio, $\Lambda_{A}=\lambda_{2}^{(A)} / M_{A}^{5}, \Sigma_{A}=\sigma_{2}^{(A)} / M_{A}^{5}$ $(A=1,2), x=\frac{1}{c^{2}}(M \omega)^{2 / 3}, \omega$ is the orbital angular velocity, and $\chi_{A}=c J_{A} / M_{A}^{2}$ is the dimensionless spin parameter of the Ath object with angular momentum $J_{A}$ (in absolute value). For simplicity, we have used $G=c=1$ units in the above equations; the form of the latter in physical units is given in Ref. [46].

As discussed in Ref. [46], there seems to exist a conceptual issue related to the inclusion of the RTLNs in the Lagrangian formulation. Since this problem is still unresolved, in the rest of the paper we shall neglect the RTLNs, setting $\tilde{\Gamma}=0$ in the GW phase.

Finally, we added Eq. (3) to the standard PN point-particle phase [31] up to 3.5PN order and up to linear order in the spin. We neglect quadratic and higher-spin corrections because they are expected to be small for NS binaries. With this choice, the point-particle phase does not depend on the spin-induced quadrupole moments of the binary components, which are quadratic in the spin and depend on the EoS.

\section{STATISTICAL ANALYSIS}

A figure of merit of GW data analysis is the matched-filter $\mathrm{SNR}, \rho$, defined through

$$
\rho^{2}=\left(d \mid h_{T}\right)=\left(h \mid h_{T}\right)+\left(n \mid h_{T}\right),
$$

where $d=h+n$ is a data stream containing a time-domain GW signal $h\left(\vec{\gamma}_{0}, \vec{\theta}_{0}\right), n$ is a given realization of the noise, and $h_{T}\left(\vec{\gamma}_{T}, \vec{\theta}_{T}\right)$ is a waveform from a given template bank. Each waveform depends upon a set of $D$-dimensional intrinsic (physical) parameters $\left\{\vec{\gamma}_{0}, \vec{\gamma}_{T}\right\}$, and upon a set of extrinsic parameters $\left\{\vec{\theta}_{0}, \vec{\theta}_{T}\right\}$ that account for angular positions, wave polarization and distance to the source. The operator $\left(h \mid h_{T}\right)$ defines the overlap between two waveforms,

$$
\left(h \mid h_{T}\right)=4 \mathcal{R} \int_{f_{\min }}^{f_{\max }} \frac{\tilde{h}(f) \tilde{h}_{T}^{*}(f)}{S_{n}(f)} d f,
$$

with $f_{\min }$ and $f_{\max }$ the lower and upper cutoff frequencies of the given detector, and $\tilde{h}$ and $S_{n}(f)$ the frequency domain representation of the signal $h$ and the noise sensitivity curve, respectively.

The extrinsic parameters are irrelevant for waveform modeling purposes since they can naturally be factored out. Then, for most of the waveform model computations, Eq. (12) is usually replaced by the normalized noise-weighted inner product or match, defined as

$$
\mathcal{M}\left(h\left(\vec{\gamma}_{0}\right), h_{T}\left(\vec{\gamma}_{T}\right)\right)=\max _{\vec{\theta}_{T}} \frac{\left(h \mid h_{T}\right)}{\sqrt{(h \mid h)\left(h_{T} \mid h_{T}\right)}}
$$

where the dependence on the extrinsic parameters is removed by (i) maximizing Eq. (12) over them and (ii) normalizing to remove the amplitude scaling. Equation (13) provides a useful tool to measure the metric distance between two waveform representations, since $\mathcal{M} \in[0,1]$, with $\mathcal{M}=1,0$ being a perfect and a zero match, respectively. In general, $\mathcal{M}$ is used as an indicator of the performance of waveform models and, for high-SNR and Gaussian noise, it may be used to provide an estimate of the systematic errors produced by the different waveform representations.

On the other hand, the parameter estimation of GW signals is based on the application of Bayesian information theory to the observed data streams. To do so, we have to compute the posterior distributions in which the data streams are matched to the waveform template banks [60],

$$
p\left(\vec{\gamma}_{T} \mid d\right) \propto p_{0}\left(\vec{\gamma}_{T}\right) \mathrm{e}^{-\frac{\left(d-h_{T} \mid d-h_{T}\right)}{2}},
$$

where $p_{0}$ is the prior distribution of the intrinsic parameters $\vec{\gamma}_{T}$. For high SNR, Gaussian noise and assuming flat priors, Eq. (14) may be substantially simplified by neglecting the noise-related factors. In this case, one can express the multivariate posterior distribution around the true parameters $\vec{\gamma}_{0}$ as (see, e.g., Appendix G of Ref. [61])

$$
p\left(\vec{\gamma}_{T}\right) \propto \exp \left(-\rho^{2}\left(1-\mathcal{M}\left(h \mid h_{T}\right)\right)\right)
$$

where we have removed the arguments to simplify the notation. The above equation allows us to describe completely the statistics in terms of the SNR and the match $\mathcal{M}\left(h, h_{T}\right)$. In other words, for a given SNR $\rho$ and a given template $h_{T}$, the mismatch $1-\mathcal{M}$ determines the probability distribution around the true values $\vec{\gamma}_{0}$. Note that the true parameters given by $\vec{\gamma}_{0}$ do not correspond to the recovered ones $\vec{\gamma}_{T}$ unless the real (injected) waveform and the template bank used are equal, $h=h_{T}$. This may insert non-negligible systematic errors that in some cases may compete in significance with the statistical ones. Thus, if we replace $h$ by a given waveform template, Eq. (15) allows us to estimate the impact of using one or another waveform template in our parameter estimation. We evaluate these effects in Sec. V, including the PN corrections described by [46].

An alternative approach is based on the Fisher-information matrix (FIM) approximation [61-63], which is known to be valid for large values of the SNR and when the noise is mostly Gaussian. In this case, it turns out that the probability of having each of the reconstructed parameters shifted by $\Delta \gamma_{i}=\left(\vec{\gamma}_{T}-\vec{\gamma}_{0}\right)_{i}$ from the real values is given by

$$
p(\overrightarrow{\Delta \gamma}) \propto \exp \left(-\frac{1}{2} \Gamma_{i j} \Delta \gamma_{i} \Delta \gamma_{j}\right)
$$

where $\Gamma_{i j}=\left.\left(\frac{\partial h_{T}}{\partial \gamma_{T i}}, \frac{\partial h_{T}}{\partial \gamma_{T} j}\right)\right|_{\vec{\gamma}_{T}=\vec{\gamma}_{0}}$ is the FIM. Then, we can compute the value of the $D$-dimensional posterior when each of 


\begin{tabular}{|c|cccccc|}
\hline fluid & $a_{0}$ & $a_{1}$ & $a_{2}$ & $a_{3}$ & $a_{4}$ & $a_{5}$ \\
\hline irrotational & -2.03 & $4.87 \times 10^{-1}$ & $9.69 \times 10^{-3}$ & $1.03 \times 10^{-3}$ & $-9.37 \times 10^{-5}$ & $2.24 \times 10^{-6}$ \\
static & -2.66 & $7.86 \times 10^{-1}$ & $-1.00 \times 10^{-2}$ & $1.28 \times 10^{-3}$ & $-6.37 \times 10^{-5}$ & $1.18 \times 10^{-6}$ \\
\hline
\end{tabular}

TABLE I. Coefficients of the fitting formula (22) describing the approximated EoS-independent relation between magnetic and electric TLNs. We consider both irrotational and static fluids [71].

the reconstructed parameters is $n \sigma$ away from the maximumlikelihood ones as

$$
p\left(\vec{\gamma}_{n \sigma}\right) \propto \exp \left(-\frac{1}{2} \Gamma_{i j} n^{2} \sigma_{\gamma_{i}} \sigma_{\gamma_{j}}\right)
$$

where $\sigma_{\gamma_{i}}=\sqrt{\Gamma_{i i}^{-1}}$ is the statistical error on the ith parameter $\gamma_{i}{ }^{3}$. Neglecting the correlation among the parameters, ${ }^{4}$ Eq. (17) reduces to

$$
p\left(\vec{\gamma}_{n \sigma}\right) \propto \mathrm{e}^{-\frac{D}{2} n^{2}}
$$

Thus, by equating Eq. (15) and Eq. (20) one gets

$$
\left(h-h_{T} \mid h-h_{T}\right) \approx 2 \rho^{2}(1-\mathcal{M}) \approx D n^{2} .
$$

The above expression allows defining the well-known distinguishability criterion between two waveform models [64]. In other words, it allows us to estimate the minimum SNR required to distinguish two waveform models within a certain $n=\Delta \gamma / \sigma$ significance, with the latter ratio equal to unity to distinguish two models with $1 \sigma$ significance, for instance. We use this definition in Sec. V as a quantitative indicator of the impact of the new terms described in the Introduction where $D=6:\left\{M, v, \chi_{1}, \chi_{2}, \Lambda_{1}, \Lambda_{2}\right\}$, where we set $n=1.64$ to get the results at the $90 \%$ credible level.

\section{IMPACT OF THE HIGHER-ORDER TIDAL TERMS IN THE GW PHASE}

The new terms considered in Sec. II B modify the waveform at high PN order. This implies that their effects gain importance as the signal approaches the high-frequency regime, possibly probing a region where current gravitational detectors are less sensitive. In general the impact of these terms will depend on the source parameters and on the merger frequency relative to the detector sensitivity. Fortunately, the parameter range of NS mergers appears to be much reduced with respect to the binary black hole case which simplifies the task of exploring the full BNS parameter space. The astrophysically relevant BNS systems are expected to have a total mass $M$ that lies in the range $[2.5,4] M_{\odot}$, the mass ratio is expected to

${ }^{3}$ A weaker requirement would be looking for the global $n \sigma$ confidence level hypersurface. Since in the FIM approximation the posterior distribution is Gaussian, this surface is a $D$-dimensional ellipsoid, and

$$
p\left(\vec{\gamma}_{n \sigma}\right) \propto \exp \left(-\frac{1}{2} r^{2}\right)
$$

where $r$ is the Mahalanobis distance

$$
r^{2}=\phi(c(n), D),
$$

with $\phi$ the inverse of the cumulative distribution of the $\chi^{2}$-distribution with be $M_{1} / M_{2} \in[1,2]$, individual spins are expected to be small ${ }^{5}$, $\chi_{1,2} \lesssim 0.05$, while the recent LIGO-Virgo constraints on the deformability parameters are $\tilde{\Lambda}<800$ at the $90 \%$ credible level [1, 6-8]. Then, before running any expensive parameterestimation analysis, we provide qualitative intuition on the importance of the terms considered in Sec. II B by showing how they affect the GW phase for different masses, mass ratios, spins, and tidal deformability coefficients.

\section{A. Relevance of the magnetic TLNs}

Let us start by discussing the magnitude of the magnetic TLN term, $\tilde{\Sigma}$, entering Eq. (3) at $6 \mathrm{PN}$ order. This term arises from the odd-parity sector of the perturbation equations $[43,50]$ thus being in principle independent from the electric TLN term. However, there exist some approximate EoSindependent relations, $\Sigma_{i}=\Sigma_{i}\left(\Lambda_{i}\right)$, that connect the magnetic TLNs to the standard electric TLNs [44]. Notice that the magnetic TLNs depend on the properties of the fluid [34, 37, 70]. In particular, the magnetic TLNs for irrotational fluids or for static fluids have the opposite sign and the quasiuniversal relation also depends on the fluid properties.

We have recently revised the properties of odd-parity perturbations and of the magnetic TLNs of a NS [71]. Our analysis confirms the discussion in Ref. [34] by showing that assuming an irrotational fluid provides a more realistic description of the fluid dynamics, since dynamical odd-parity perturbations enforce irrotationality in the stationary limit [71]. Nevertheless, for the sake of completeness, here we shall consider both cases and we use the following fitting formula

$$
\log \left( \pm \Sigma_{i}\right)=\sum_{n=0}^{5} a_{n}\left(\log \Lambda_{i}\right)^{n}
$$

where $i=1,2$ for the two bodies, and the plus or minus sign is for static or irrotational fluid, respectively. The form of the above fit is the same as that of Ref. [44], whereas the coefficients have been computed in Ref. [71] and are given in Table IV A for the cases of irrotational and static fluids, respectively. Our results agree with those of Ref. [37] and with the revised ones in Ref. [44] in the relevant regimes [71]. We also checked that the analysis presented below is insensitive to the small differences between different fitting functions.

$D$ degrees of freedom and $c(n)$ the probability of falling inside the $n \sigma$ confidence region $(c(1) \sim 0.68, c(2) \sim 0.95$, etc.).

${ }^{4}$ This assumption is justified by the fact that we are interested only in the weighted-tidal deformability $\tilde{\Lambda}$ parameter, which at so high SNR is weakly correlated to the other parameters (cf. Sec. V).

5 Although the distribution of NS spins is uncertain, old NSs in the late stages of a binary inspiral are expected to rotate rather slowly. The fastest spinning NS observed so far in a compact system is the most massive component of 
Equation (22) provides a mapping from $\Lambda_{1,2}$ to $\Sigma_{1,2}$ which is accurate at the level of a few percent [37, 44, 71]. Using this relation allows one to remove the explicit dependence on the magnetic TLNs in the waveform. In this way the final phase (3) depends only on the electric TLNs $\Lambda_{1,2}$, on the masses $M_{1,2}$, and on the spins $\chi_{1,2}$.

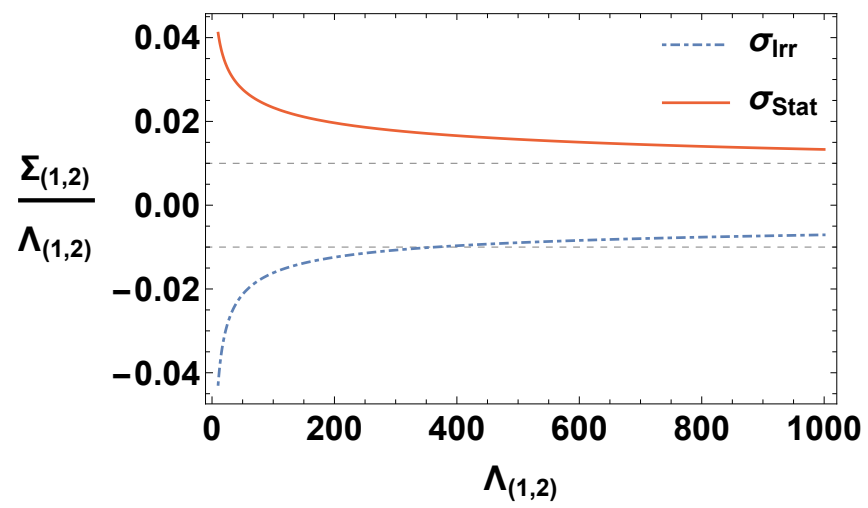

FIG. 1. Approximated universal relations between the electric $\left(\Lambda_{i}\right)$ and the magnetic $\left(\Sigma_{i}\right)$ quadrupolar TLNs as proposed in [44]. The dashed lines define the points in the parameter space where the magnetic TLN contribution reaches $1 \%$ with respect to standard electric TLNs. The magnetic TLNs depend on the properties of the fluid [34, 37, 70]; we show the cases of irrotational and of static fluids.

In Fig. 1 we show the approximate EoS-independent relations between the (quadrupolar) electric and magnetic TLNs for irrotational and static fluids. For typical values of the compactness of a NS, the ratio $\Lambda_{i} / \Sigma_{i} \approx \pm 100$, where the plus and minus signs refer to static and irrotational fluids, respectively. This anticipates that the 6PN order coefficient in the tidal phase is dominated by the next-to-leading corrections proportional to $\Lambda_{1,2}$, rather than by the terms proportional to $\Sigma_{1,2}$ that enter at the same PN order. As shown in Fig. 1, the ratio $\Sigma_{i} / \Lambda_{i}$ depends only mildly on $\Lambda_{i}$ and, when $\Lambda_{i} \gtrsim 200$, the relation $\Sigma_{i}\left(\Lambda_{i}\right)$ is approximately linear. As already mentioned, assuming static or irrotational fluid yields opposite magnetic corrections to the tidal phase. Static fluids yield magnetic TLNs that appear with the same global sign as the $\Lambda_{i}$ and the $\delta \Lambda$ terms (i.e., attractive tidal effects in the two-body dynamics). This would (slightly) increase the overall impact of the tidal effects at the next-to-leading order. On the other hand, the more realistic case of irrotational fluid yields magnetic TLNs that act in the opposite direction (repulsive tidal effects), thus inducing a partial screening of the subleading tidal terms. This qualitative analysis suggests that neglecting the magnetic contribution may lead to a (small) bias in the estimate of $\Lambda_{i}$ that will be of opposite sign depending of the type of fluid considered. We explore the relevance of these effects in Sec. V.

the double pulsar system PSR J0737-3039A [65], with a spin period of $\approx 23 \mathrm{~ms}$, which corresponds to $\chi \sim 0.02-0.05$, depending on the EoS [66, 67] ( $\chi \sim 0.02$ for APR [68] EoS). Such a rotation rate is not expected to decrease substantially as this system approaches the merger (see Ref. [69] for a discussion). On the other hand, the observation of numerous isolated millisecond pulsars suggests that spin rates as high as $\chi \sim 0.1$ [69] might be found also in BNS systems.

\section{B. Relevance of the spin-tidal couplings}

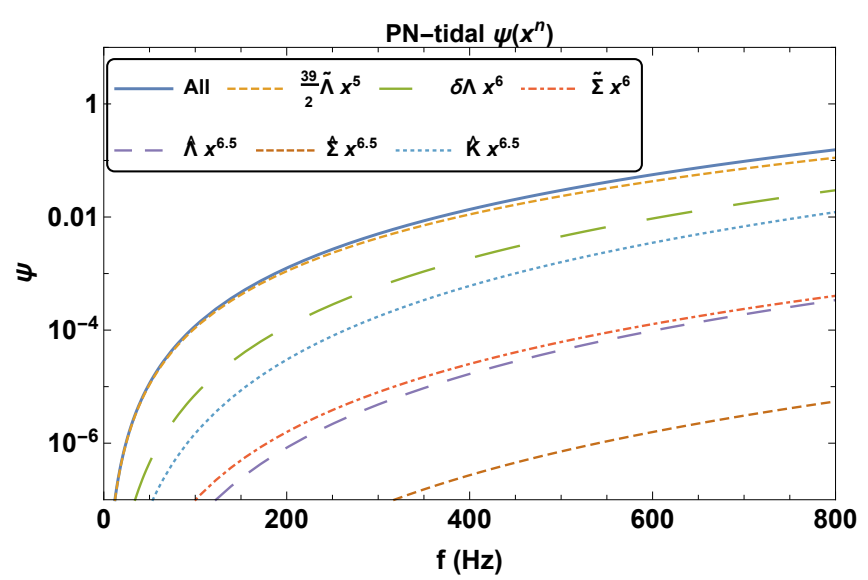

FIG. 2. Each of the tidal contributions from Eq. (3) to the GW phase (in absolute value) as a function of the GW frequency. We considered an equal-mass binary with total mass $2.8 M_{\odot}$, with $\Lambda_{1}=\Lambda_{2}=300$, and spins $\chi_{1}=\chi_{2}=0.05$. The contribution from the spin-tidal couplings at $6.5 \mathrm{PN}$ order $\hat{\Lambda}$ scales linearly with the spin.

In Fig. 2 we present the individual contributions to the GW phase of each of the tidal terms described in Eq. (3) along the relevant LIGO-Virgo frequency domain. To do so, we consider a system with physical parameters roughly compatible with GW170817: an equal-mass binary with total mass $2.8 M_{\odot}$, electric TLNs $\Lambda_{1}=\Lambda_{2}=300$, and spin parameters $\chi_{1}=\chi_{2}=0.05$, in order to show the effects of the $6.5 \mathrm{PN}$ order spin-tidal coupling terms $\hat{\Lambda}$. As expected, the leadingorder 5PN order term $\tilde{\Lambda}$ dominates the GW tidal phase by more than an order of magnitude with respect to higher-order terms. The next term in order of importance is the 6PN orderelectric term $\delta \Lambda$, which contributes on average around $20 \%$ of the total tidal phase evolution. Indeed, this was the highest PN tidal term accounted for in the analysis of GW170817 [1].

The next term in order of relevance is the $6.5 \mathrm{PN}$ order tidal tail $\hat{K}$, whereas the spin-tidal term $\hat{\Lambda}$ is significantly less dominant: its relative contribution is smaller than the total tidal phase by about 2 orders of magnitude and also contributes about $3 \%$ with respect to the total $6.5 \mathrm{PN}$ order coefficient. This suggests that it might be safely neglected for binaries with $\chi_{i} \approx 0.05$. On the other hand, this term grows linearly with the spin so that it might become important if highly spinning NS binaries exist in nature and for high SNR scenarios. Finally, the lowest contributions come from the magnetic TLNs [50], which agree with the estimates obtained by [28]. This has to do with the small ratio between the magnetic and the electric TLNs shown in Fig. 1.

We quantify the above expectations in Sec. V. We note that the higher-order tidal terms have a simple dependence across the parameter space of the binary. Thus, they can easily be computed for different values of $\Lambda_{i}, M_{i}$, and $\chi_{i}$.

For example, we can explore the ratio of the dominant 5PN6PN order factors $\tilde{\Lambda}$ and $\delta \Lambda$ as a function of the total mass $M$ and the mass ratio $v$. We show this in Fig. 3 for a GW170817like event with $\Lambda_{1}=\Lambda_{2}=300$. The $6 \mathrm{PN}$ order terms $\delta \Lambda$ do not contribute by more than $20 \%$ relative to the leading-order term in the whole parameter space. We observe that the parameter that mostly affects this ratio is the total mass $M$, being the mass ratio subdominant. As we explain in the next lines, 


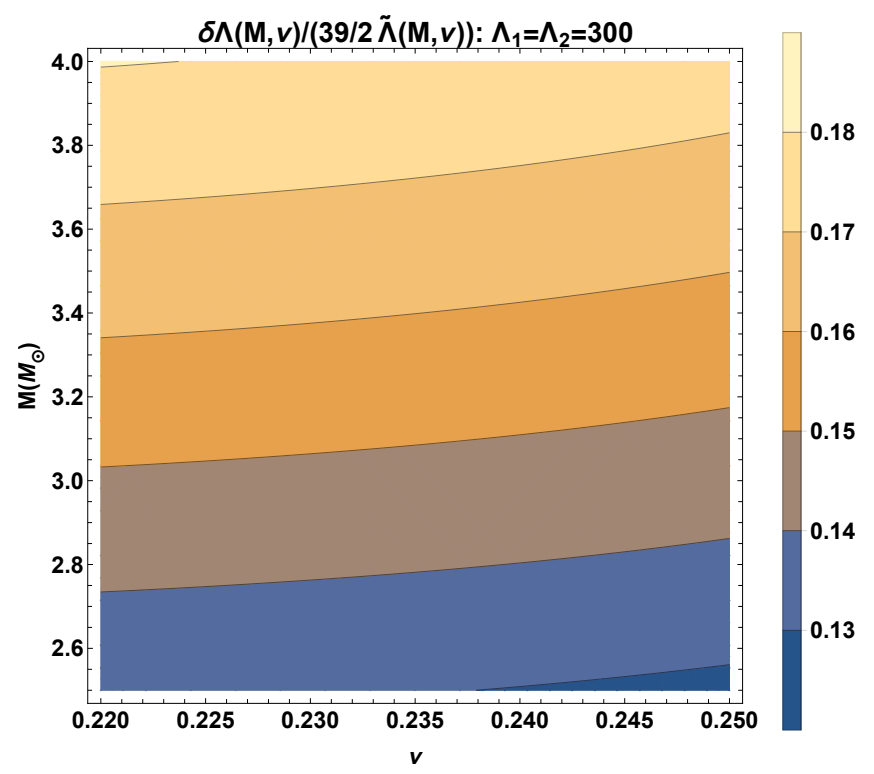

FIG. 3. Absolute value of the ratio between the leading-order and the next-to-leading order coefficients, $2 / 39 \delta \Lambda / \tilde{\Lambda}$, at $f=300 \mathrm{~Hz}$ as a function of the total mass $M$ and the mass ratio $v$. We set $\Lambda_{1}=\Lambda_{2}=$ 300, consistent with GW170817 [1, 6-8].

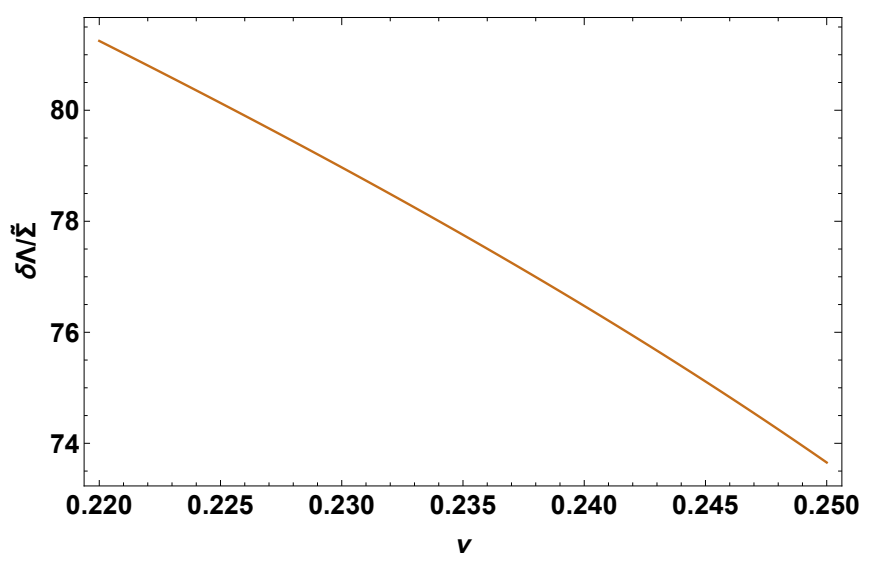

FIG. 4. Ratio (in absolute value) between the two next-to-leading order contributions, $\delta \Lambda / \tilde{\Sigma}$, as a function of the symmetric mass ratio $v$ for $\Lambda_{1}=\Lambda_{2}=300$ and for irrotational fluids. Notice that the dependence on the total mass scales out since we are comparing equal-order coefficients.

this ratio increases linearly with $\tilde{\Lambda}$. This implies that, considering the recent constraint $\tilde{\Lambda} \lesssim 800[1,6]$, this ratio could increase by a factor $\sim 3$, thus allowing for a maximal contribution of about $40 \%$ in ultrahigh mass $\left(4 M_{\odot}\right)$ NS mergers and of about $30 \%$ in ordinary binaries.

In addition, in Fig. 4 we show the ratio between the coefficients $\delta \Lambda$ and $\tilde{\Sigma}$ for a $\Lambda_{1}=\Lambda_{2}=300$ binary. The ratio $\delta \Lambda / \tilde{\Sigma}$ does not depend strongly on the mass ratio (since we are comparing PN terms at the same order the total mass scales out), thus being dominated by the different scale between the electric and magnetic TLNs.

\section{RESULTS}

To quantify the effect of the 6PN order magnetic TLNs and the spin-tidal 6.5PN order contributions, we adopt an analysis based on the FIM introduced in Sec. III. In particular, our analysis is valid for high-SNR and Gaussian noise. As we shall verify a posteriori, the former assumption is anyway necessary, since the effect of higher-order tidal terms is typically small.

We take the only BNS event observed so far by the LIGOVirgo Collaboration, GW170817, as a reference. This event, observed with a SNR of $\rho=32.4$, was consistent with a BNS system with masses compatible with $M_{1} \sim M_{2} \sim 1.4 M_{\odot}$, with spins of the components compatible to zero, $\chi_{1,2} \sim 0$, and the $90 \%$ credible intervals on the combined electric tidal deformability $\tilde{\Lambda}$ have been recently constrained to lie within $\sim[70,800][7,8]$ with the median value being $\tilde{\Lambda}=300$. Though the observation of such a high SNR event was rather unlikely considering the previous event rate predictions [72], the inclination reported by the LIGO-Virgo Collaboration [1] allows for a nonzero inclination angle with respect to the Earth's observation line $\left(i \sim 30^{\circ}\right)$. This could have been reduced by a factor of approximately 1.15 the total SNR of this event relative to the SNR that the same source would have produced if oriented face on (see, e.g., [73]). Based on the above discussion, we consider two different scenarios: the standard scenario where the physical parameters are taken to be those compatible with GW170817, that is, $\rho=32.4, M_{1}=$ $M_{2}=1.4 M_{\odot}, \tilde{\Lambda}=300$; and a more optimistic scenario in which we consider the hypothetical case of observing an event with the physical parameters compatible with GW170817, but in a face-on orientation, i.e., with $\rho=37,{ }^{6}$ and fixing the maximum value allowed by the LIGO-Virgo posterior distributions. The optimistic scenario maximizes the detectability of the tidal effects considered in this work. Finally, we consider two detectors: (i) LIGO in its O1 configuration [74], known as eaLIGO, with $\left\{f_{\min }, f_{\max }\right\}=\{23,2048\} \mathrm{Hz}$; and (ii) the planned third-generation detector Einstein Telescope (ET) [75-77] in its ET-D configuration ${ }^{7}$ with $\left\{f_{\text {min }}, f_{\text {max }}\right\}=$ $\{1,2048\} \mathrm{Hz}$, for which current prospects anticipate a sensitivity gain of factor $\approx 45$ compared to eaLIGO. The correcting factors to translate a LIGO-Virgo event to an ET one are described in Appendix A. ${ }^{8}$

\section{A. Impact of 6PN order magnetic terms on TaylorF2 approximants}

Systematic errors on GW parameter estimation are induced by the incompleteness of the waveform template banks. This may produce an artificial bias with respect to the true parameters that in some cases may overtake the statistical uncertainty driven by the dectector's noise. Here we evaluate the impact of neglecting the 6PN order magnetic coefficient $\tilde{\Sigma}$ by comparing three different sets of analytic waveforms. As previously discussed, we consider each magnetic TLN $\Sigma_{i}$ to be related to the electric $\Lambda_{i}$ through the universal relations shown in Table IV A. Then, we explore the possibility of observing two simulated GW triggers, tagged as $h_{s t}$ and $h_{i r r}$, that are match

\footnotetext{
${ }^{6}$ This factor is easily obtained from the ${ }_{-2} Y^{22}(i=30, \phi)$ spherical harmonic.

7 The $D$ stands for the so-called xylophone configuration that is expected to be more sensitive at low frequencies than other alternative ET designs as the V-shaped configuration ET-B [76, 78].

${ }^{8}$ The geometrical factors to transform from a LIGO-type observatory to ET are explained in [78, 79].
} 
filtered with a waveform template bank $h_{0}$. The triggers and the template are defined as

- $h_{0}$ : TaylorF2 waveform template bank given by Eq. (3), truncated at 6PN order, and with vanishing magnetic TLNs, i.e. $\tilde{\Sigma}=0$, as in $[1,7,8]$;

- $h_{s t}$ : GW signal consistent with a TaylorF2 model truncated at 6PN order, with magnetic TLNs included assuming a static fluid;

- $h_{\text {irr }}$ : GW signal consistent with a TaylorF2 model truncated at 6PN order, with magnetic TLNs included assuming an irrotational fluid.

To produce the posterior distribution (15), we first need to compute the match (13) between our magnetic GW signals $h_{\text {st }, \text { irr }}$ and our reference waveform template bank $h_{0}$. We set the masses and spins of the GW signals $h_{s t, i r r}$ and the template bank waveforms $h_{0}$ to $M_{1}=M_{2}=1.4 M_{\odot}$ and $\chi_{1}=\chi_{2}=0$, respectively. In addition, we consider two possible values (injections) $\tilde{\Lambda}_{0}=300,800$ for the tidal deformability of our simulated GW events $h_{s t, i r r}$, consistent with our standard and optimistic scenarios. We also assume that the two NSs are described by the same EoS so that, since $M_{1}=M_{2}$, we have $\Lambda_{1}=\Lambda_{2}$ and $\Sigma_{1}=\Sigma_{2}$. Then, by varying $\Lambda_{1}$ such that $\tilde{\Lambda} \in[0,2000]$, we generate a $\tilde{\Lambda}$-dependent distribution for the match $\mathcal{M}\left(h_{0}(\tilde{\Lambda}) \mid h_{s t, i r r}\left(\tilde{\Lambda}_{0}\right)\right)$, that is translated to $p(\tilde{\Lambda})$ by means of Eq. (15). We repeat the analysis for eaLIGO and ET-D noise sensitivity curves.

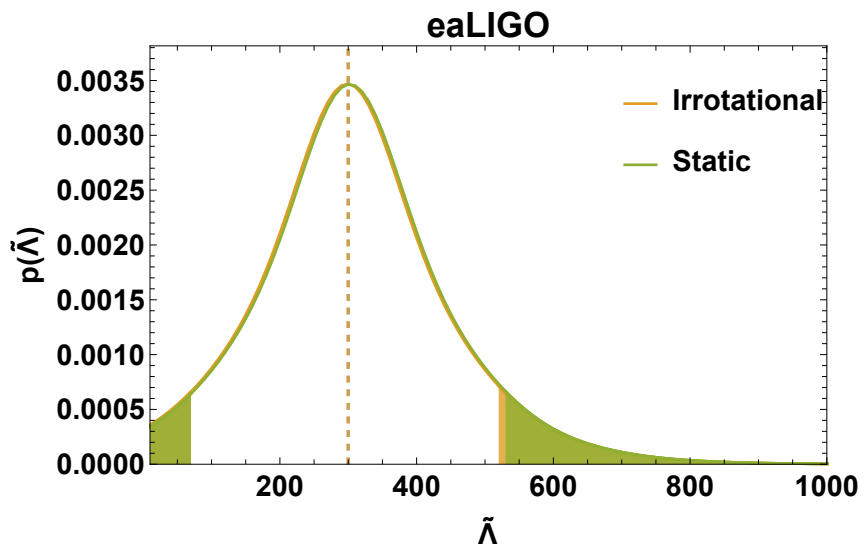

FIG. 5. Probability distributions resulting from the match between $h_{0}$ and $h_{s t, i r r}$ for an injected value $\tilde{\Lambda}_{0}=300$ and $\rho=32.4$. The offset produced by neglecting the magnetic TLNs, both static and irrotational, is shown to be negligible for events compatible with GW170817 and the eaLIG0 sensitivity curve. The solid area defines the region out of the $90 \% \tilde{\Lambda}$ credible intervals.

In Fig. 5 we show the probability distributions obtained in our standard scenario by matching our two GW events $h_{s t, i r r}$ to the waveform template bank $h_{0}$. We observe that the posterior distributions obtained for $h_{s t, i r r}$ are compatible with the injected value $\tilde{\Lambda}_{0}=300$, thus not revealing any sensitive offset with respect to the zero-magnetic model $h_{0}$. The differences on the peaks obtained for static (green) and irrotational (orange) fluids are on the order of $\left|\tilde{\Lambda}_{0}-\tilde{\Lambda}_{s t, i r r}\right| \sim 1$, where the solid area determines the $90 \%$ credible intervals. This is consistent with the results of Fig. 2, where the $\tilde{\Sigma}$ contribution appears to be even more subdominant than the 6.5PN order tidal-tail effect. Moreover, considering that these effects do not vary significantly in the unequal-mass case (Fig. 4) and

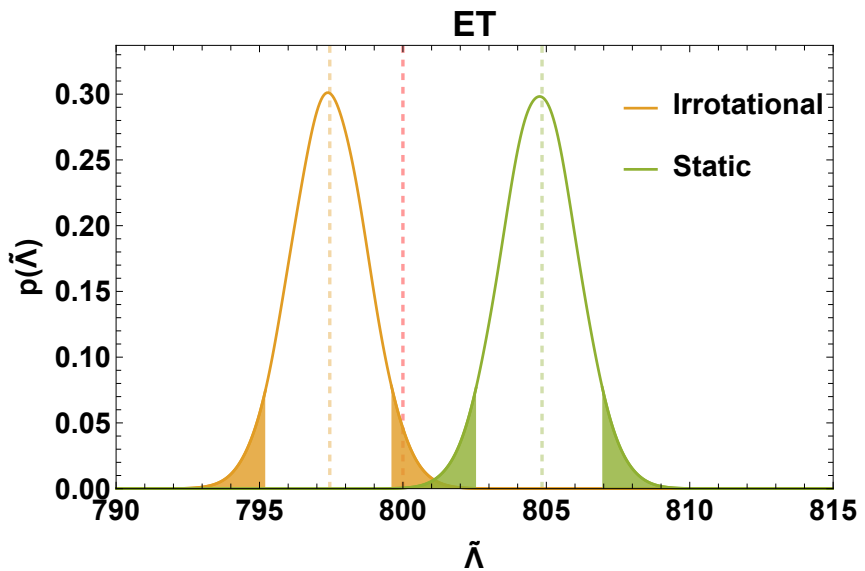

FIG. 6. Same as in Fig. 5 but for our optimistic scenario and assuming a detection with ET-D. The vertical dashed line in red corresponds to the position of the injected value $\tilde{\Lambda}$, while the orange and the green ones correspond to the peak value for irrotational and static fluids, respectively. The solid areas (orange and green) define the region out of the $90 \%$ credible intervals.

that their dependence on the total mass is exactly zero (Fig. 4), we do not expect to observe any significant gain for a different point across the parameter space. Therefore, these results suggest that the effect of magnetic TLNs is negligible for the measurement of the tidal deformability and that measuring the magnetic TLNs independently with eaLIGO will be very unlikely. Unfortunately, this also prevents the constraint of the dynamical properties of the NS fluid (i.e., static versus irrotational) according to a putative measurement of the sign of $\Sigma_{i}$. We note that if these terms are negligible within our simplified analysis (in which the only parameter that is varied is $\tilde{\Lambda}$ ), they would be even more difficult to measure within a rigorous and more expensive multidimensional Bayesian analysis performed on $h_{s t, i r r}$.

Finally, we can also carry out the same analysis in our optimistic scenario (that is, $\tilde{\Lambda}=800$ ) and assuming the ET-D noise sensitivity curve. This gives an SNR larger than in the standard scenario by roughly a factor of 55 ; i.e., we set $\rho=1750$, which comes from observing GW170817 with ET-D (see Appendix A). From Eq. (15), in the large- $\rho$ limit the FIM errors scale as $\sigma_{i} \propto 1 / \rho$. This implies that any gain on the SNR will sharpen our posterior distributions around the best-likelihood values that, in general, will be different for different waveform approximants. Thus, in the optimistic scenario the offset between the recovered tidal deformability $\tilde{\Lambda}$ given by $h_{s t, i r r}$ and the injected value $\tilde{\Lambda}_{0}$ should be larger than in the standard scenario assuming a GW170817-like detection with LIGO-Virgo.

In Fig. 6 we show the posterior distributions generated when assuming static and irrotational fluids. Notice that the displacement between distributions is larger than in the standard scenario, as well as the $90 \%$ credible levels delimited by the orange and green solid areas. The sign of the offset is directly correlated with the sign of the magnetic TLNs. In the case of irrotational fluids, the magnetic TLNs enter at lower order than (but with the sign opposite to) the electric TLNs, thus tending to decrease the tidal effects. This induces a small underestimation of $\tilde{\Lambda}$ with respect to the injected value $\tilde{\Lambda}_{0}$, the differences being larger as one increases the injected value $\tilde{\Lambda}_{0}$. The opposite happens for static fluids: in this case the sign of electric and magnetic TLNs is the same, leading to an 
increase of the tidal effects, and thus inducing a small overestimate of $\tilde{\Lambda}$. The offset between the distributions is sufficient to place the peak values outside of the credible regions. In other words, the differences may be marginally observable as long as the optimistic scenario is considered. Therefore, based on the above analysis, we can estimate that the error induced by not including tidal-magnetic effects in current waveform models [13, 55] (both NR-calibrated and analytical ones) will not be observed for the next BNS observations with $2 \mathrm{G}$ gravitational wave detectors, but they may produce a not negligible impact on $3 \mathrm{G}$ detectors such as ET.

\section{B. Impact of 6.5PN order terms on TaylorF2 approximants}

Following the discussion of the previous section, let us now consider the 6.5PN order terms, i.e., the tidal-tail term and the tidal-spin coupling. We quantify the magnitude of these terms by performing the same match/distinguishability analysis previously discussed, but now considering a spinning binary. For simplicity, and because they are more realistic [34, 71], we only consider the magnetic TLNs arising from an irrotational fluid. ${ }^{9}$ For the present analysis, the set of waveforms considered are the following:

- $h_{0}$ : 6PN order TaylorF2 waveform template bank, with zero spin-tidal contributions and setting to zero the tidal-tail contribution $\hat{K}=0$;

- $h_{\chi}$ : GW signal described by a $6.5 \mathrm{PN}$ order TaylorF2 waveform accounting for irrotational fluids, with nonvanishing spins and $\hat{K}=0$.

Note that in both cases we are not considering the tail-tidal term, imposing $\hat{K}=0$. The reason is that the tail-tidal term limits the convergence domain of the 6.5PN order term to frequencies $f \lesssim 100 \mathrm{~Hz}$. Beyond these frequencies, including the 6.5PN order tail contribution makes the accuracy of the $\mathrm{PN}$ series worse than that retaining only terms up to 6PN order (see Fig. 10 below). We discuss this issue in detail in Sec. VI.

Also note that, since the spin-tidal terms are linear in the spin and since we are neglecting quadratic and higher-spin terms in the point-particle phase, the entire PN phase is symmetric under spin inversion, $\chi_{i} \rightarrow-\chi_{i}$.

In Fig. 7 we show the effect of the spin-tidal corrections with respect to the standard 6PN order approximant for the ET-D noise sensitivity curve. The injected parameters are consistent with an equal-mass binary with total mass $M=2.8 M_{\odot}$, equal spins $\chi_{1}=\chi_{2}=0.05$, and $\tilde{\Lambda}_{0}=300$, thus setting a conservative (standard) scenario where the spins are compatible with current astrophysical observations. Note that the probability distributions match almost perfectly the nonspinning predictions described by $h_{0}$ though the spin-tidal effects tend to induce a minimal shift on $p(\tilde{\Lambda})$ that depends on the sign of the spin. The impact on the recovery of $\tilde{\Lambda}$ is small, not producing a bias larger than $1 \%$ with respect to $\tilde{\Lambda}_{0}$. The sign of the offset tends to overestimate and underestimate $\tilde{\Lambda}$ for positive (dashed green line) and negative (orange line) spins, respectively. This can be explained intuitively by observing

\footnotetext{
${ }^{9}$ At any rate, the contribution of $\hat{\Sigma}$ is much smaller than that of $\hat{\Lambda}$, since $\Sigma_{i} \approx \Lambda_{i} / 100$.
}

the relation between the electric $6.5 \mathrm{PN}$ order spin-tidal coefficient $\hat{\Lambda}$ and the $6 \mathrm{PN}$ order $\tilde{\Sigma}$ one from Fig. 2. We observe that for $\chi_{1}=\chi_{2}= \pm 0.05$ the corrections induced by these terms produce similar corrections to the orbital phase, where the role of positive spins would be similar to that of static fluids while negative spins would affect similarly to irrotational fluids. Moreover, notice the similarity between the offsets obtained in Figs. 6 and 7, with the corrections on the former being larger due to the larger $\tilde{\Lambda}_{0}$ considered. Thus, we see that the linear dependence of the orbital phase on each of the tidal contributions induces a similar linear behavior on the bias produced when comparing different approximants. Thus, Fig. 7 shows that the spin-tidal coefficients for an event fully compatible with GW170817 are negligible even when assuming a detection with ET-D. This is also in agreement with the results obtained by full hydrodynamical NR simulations of BNS systems [13], where the spin-tidal effects do not show any significant contribution to the orbital phase for spins as high as $\chi \sim 0.15$. The picture slightly improves when we compute

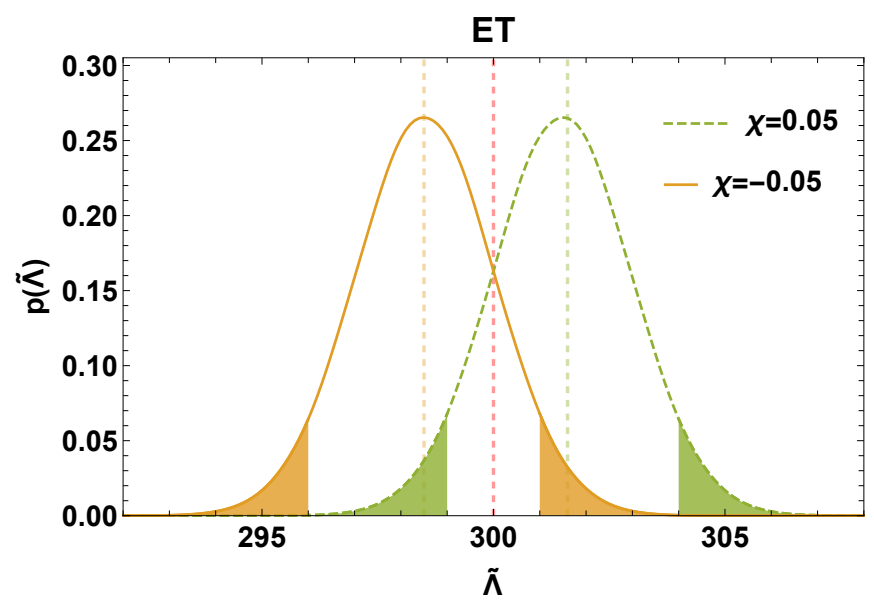

FIG. 7. Probability distributions obtained in our standard scenario for a binary spinning at $\chi=0.05$ (dashed green line) and $\chi=-0.05$ (orange line), assuming irrotational fluids, with the ET-D noise sensitivity curve. The vertical dashed lines define the best-likelihood values while the solid areas define the $90 \%$ credible intervals. The red dashed vertical line defines the injected value $\tilde{\Lambda}_{0}$.

the deviations in our optimistic scenario, that is, increasing $\rho$ by a factor of 1.15 and setting $\tilde{\Lambda}_{0}=\{300,800\}$, but now also considering spin rates as high as $\chi_{1}=\chi_{2}= \pm 0.1$ in order to maximize the spin-tidal effects. The results for this case are shown in Fig. 8. For $\tilde{\Lambda}=300$ the best-likelihood values for $\tilde{\Lambda}$ for both aligned (orange line) and antialigned (dashed green line) binaries lie approximately on the tails of the $90 \%$ credible intervals delimited by the solid areas, thus being the spintidal waveforms $h_{\chi}$ marginally distinguishable from the template $h_{0}$. The peak offsets go in the same direction as in Fig. 7 but now largely increased because the SNR, the electric TLN $\tilde{\Lambda}$, and the spins $\chi_{1,2}$ for the simulated events $h_{\chi}$ are a factor of $\sim 2.7,2$, and 55 times larger, respectively (see Appendix A). In the pure optimistic scenario, that is, $\tilde{\Lambda}=800$, we observe a much larger offset with respect to the small $\tilde{\Lambda}$ case of $6 \sigma$ and thus clearly placing the injected value outside the $90 \%$ credible intervals. Therefore, a spin-tidal model would produce a distribution substantially different from the pure 6PN models being its effects relevant for parameter estimation.

Finally, we also provide an estimate of the minimum values 

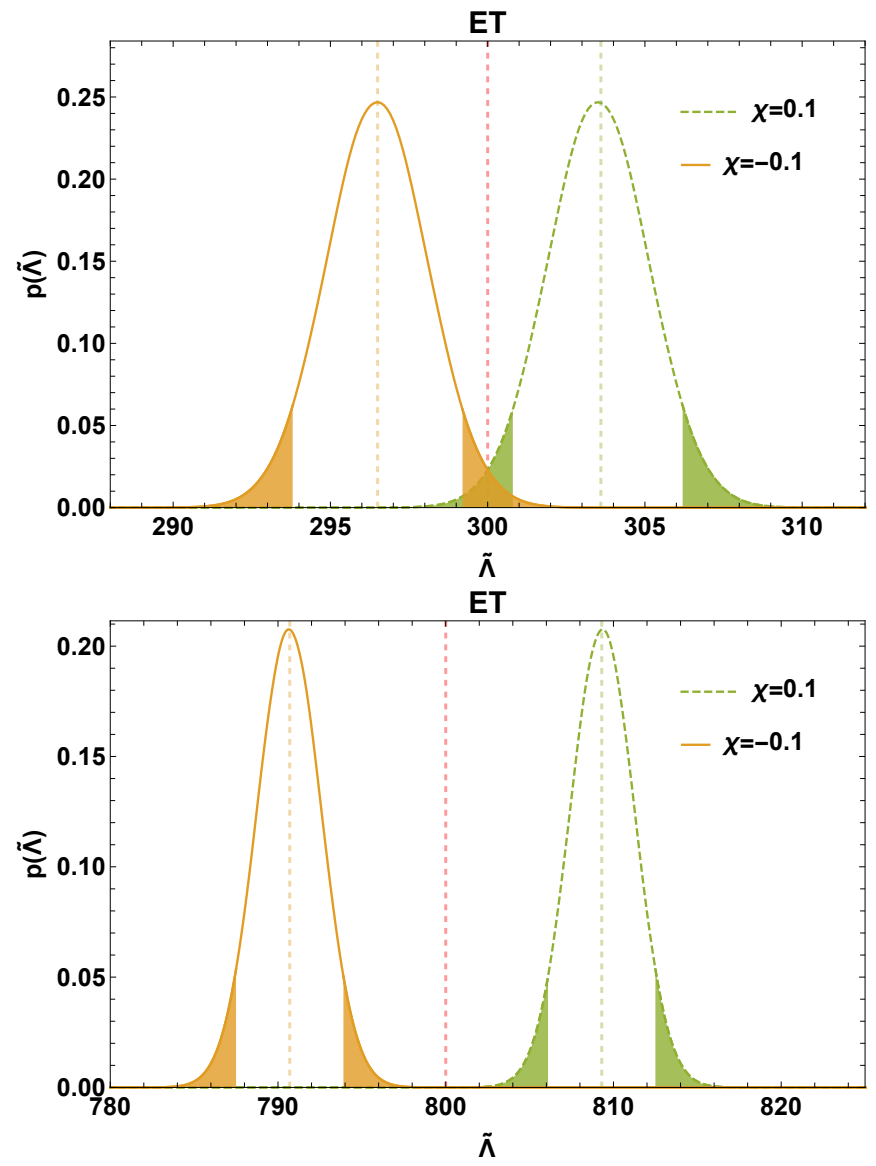

FIG. 8. Probability distributions obtained for two BNS spinning at $\chi=0.1$ (dashed green line) and $\chi=-0.1$ (orange line), assuming irrotational fluids, with the ET-D noise sensitivity curve but now considering the case of observing GW170817 with an optimal orientation and $\tilde{\Lambda}=\{300,800\}$ (top and bottom panels). The vertical dashed lines define the best-likelihood values while the solid areas define the $90 \%$ credible intervals. The red dashed vertical line defines the injected value $\tilde{\Lambda}_{0}$.

for the triplet $\chi-\rho-\tilde{\Lambda}_{0}$ (with $\chi_{1}=\chi_{2}=\chi$ ) required to distinguish the effects of the spin-tidal terms for a GW170817like event detected with ET-D. To do so, we have computed the match of a $h_{0}\left(\tilde{\Lambda}_{0}, \chi\right)$ against $h_{\chi}\left(\tilde{\Lambda}_{0}, \chi\right)$, for $\tilde{\Lambda}_{0} \in[0,2000]$ and $\chi \in[-0.79,0.79]$. The results of the match are translated to $\rho$ through Eq. (21) for a $D=6$ parameter space, where we require one to estimate all the parameters at $90 \%$ credible level, i.e., $n=1.64$. The results of this analysis are shown in Fig. 9. The contour lines represent the minimum SNR needed to observe some characteristic combination of $\tilde{\Lambda}_{0}$ and $\chi_{1}=\chi_{2}=\chi$. The solid and dashed vertical grid lines $\tilde{\Lambda}_{0}=\{300,800\}$ set the median and $90 \%$ upper limit provided by $[1,8]$, respectively. Then, the intersection of $\tilde{\Lambda}_{0}=800$ with the $\rho=\{1500,1750\}$ contours shows that the minimum spin required to distinguish the spin-tidal effects from a $h_{0}$ template at the $90 \%$ level is $\chi \sim \pm 0.07$, respectively. Notice that the intersection of the $\tilde{\Lambda}_{0}=800$ line with the green contour line $(\rho=1750)$ corresponds to the particular case shown in Fig. 8. Moreover, from Fig. 9, we see that larger spins are required to attain the same SNR as $\tilde{\Lambda}_{0}$ decreases. In particular, for $\tilde{\Lambda}=300$ and $\rho=1750$ the intersection occurs at $\chi \sim \pm 0.15$. Therefore, spin-tidal couplings are only expected to affect significantly the signal for putative optimally oriented BNS events, observed with third-generation detectors,

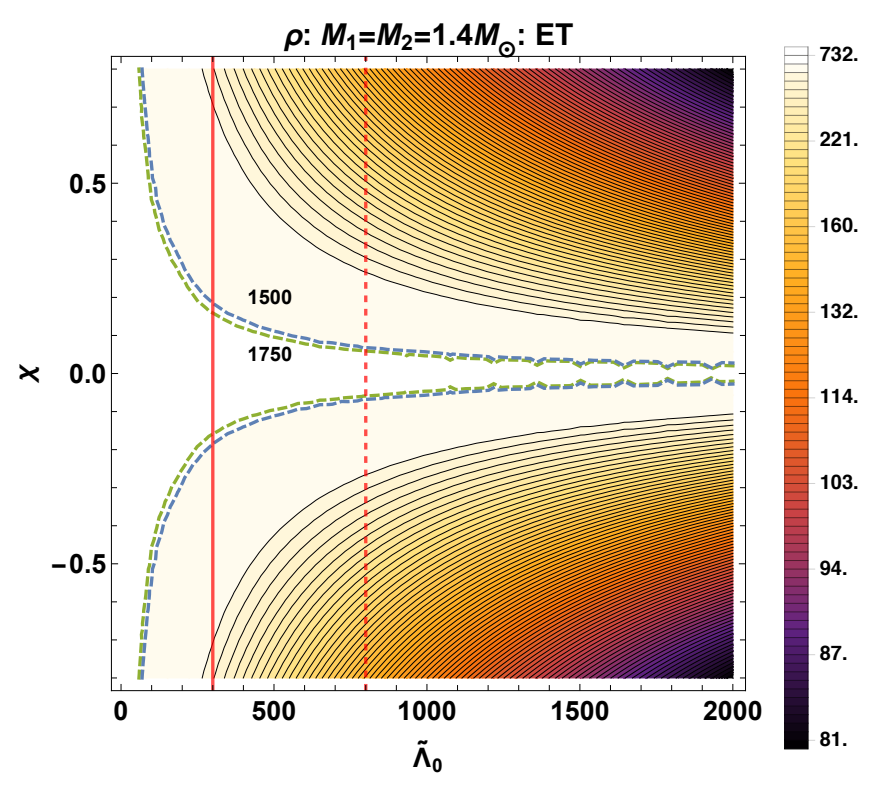

FIG. 9. Estimation of the SNR required to distinguish the effects of the spin-tidal terms considering the ET-D noise sensitivity curve. The vertical red grid lines fix the tidal deformabilities consistent with the median and $90 \%$ upper limits provided by LIGO-Virgo $[1,8]$ respectively. The blue and green contour lines correspond to the SNR of our standard and optimistic scenarios.

and for moderately large spins. On the other hand, the calibration of these effects on current waveform templates would have a non-negligible impact only if high-spin binaries (with $\left.\chi_{i} \gtrsim 0.1\right)$ evolve and merge in our local universe.

Finally, we note that we have also estimated the onedimensional probability distributions on $p(\tilde{\Lambda})$ by running a six-dimensional Markov chain Monte Carlo algorithm on Eq. (15), where $p(\tilde{\Lambda})$ is obtained by marginalization. By doing so, we did not observe any relevant differences with respect to the distributions $p(\tilde{\Lambda})$ obtained in this section, thus suggesting that the correlations between the physical parameters do not affect our results in such high SNR scenarios.

\section{TRUNCATION EFFECTS ON HIGH-PN ORDER TAYLORF2 TERMS}

PN models approximate the orbital dynamics by a powerseries expansion of the equations in terms of the parameter $x=v^{2} / c^{2}=(G \omega M)^{2 / 3} / c^{2} \ll 1$. However, in the highfrequency regime the optimal truncation order may be limited by the convergence properties of the PN series. This has been extensively studied in the case of binary black holes, where the expansion above 3PN order is shown to break down at relatively low frequencies [80-83]. In this section we study the properties of the tidal part of the PN series as an asymptotic series [84]. Formally, a power series is said to be asymptotic to a function $f(x)$ as $x \rightarrow x_{0}$ if for each $N$

$$
f(x)-\sum_{n=0}^{N} a_{n}\left(x-x_{0}\right)^{n} \ll a_{N}\left(x-x_{0}\right)^{N} .
$$

This equation states that, to satisfy the asymptotic condition near some point $x_{0}$, the difference between a function and the $N$-truncated sum of the series should be much smaller than 
the last term kept in the expansion. If the series is divergent [or it is not converging to $f(x)$ ], for each given point $x$ there is a maximum order $N=N(x)$ for which the match between the function and the series truncated up to that order is the optimal one, which means that including higher-order terms will decrease the accuracy of the approximation. For the PN case Eq. (23) reduces to

$$
\psi(x)-\sum_{n=0}^{N} a_{n / 2} x^{n / 2} \ll a_{N / 2} x^{N / 2}
$$

where $2 n$ is the PN order [see Eq. (3)] and the function $\psi(x)$ is the exact (but unknown) full gravitational waveform phase for the binary under consideration. Likewise, the range of validity of the truncated expansion at some fixed PN order can be limited to some maximum point $x=x_{\max }$, above which Eq. (24) is no longer satisfied. The exact value of $x_{\max }$ will in general vary across the parameter space (component masses, spins, etc.) though for BNS systems we expect this variation to be smaller than for binary black holes due to the relative smallness of the parameter space.

Here we study the asymptotic behavior of the PN tidal terms in the case of a nonspinning, equal-mass BNS with vanishing magnetic TLNs. The latter approximation should not affect our analysis since, as shown in the previous sections, magnetic TLNs give a negligible contribution to the GW phase. Then, the tidal approximants can be considered valid for parameter estimation studies if and only if all these terms satisfy Eq. (24), across the full parameter space and along the full frequency regime of ground-based detectors.

To illustrate this, we assume the NR-calibrated model IMRPhenomD-NRTidal [13] as the true underlying tidal part of GW phase $\psi(x)$ in Eq. (24), recalibrating its coefficients to also recover the $6.5 \mathrm{PN}$ order tail coefficient $\hat{K}$ in the lowfrequency limit. Doing so we ensure that the model by itself represents effectively the same phase evolution as the original one but achieving a better match with the PN solutions at intermediate frequencies. Then, we check whether Eq. (24) is satisfied for all the truncated expansions of the TaylorF2 approximant previously considered. For this analysis we adopt the physical parameters of GW170817; in particular we consider nonspinning binaries. In Fig. 10 we show the quantity

$$
\Delta(x)=\left|\psi(x)-\sum_{n=0}^{N} a_{n / 2} x^{n / 2}\right|
$$

[i.e.,the left-hand side of Eq. (24)] for each PN tidal order, namely $\left\{5 \mathrm{PN}, 6 \mathrm{PN}, 6.5 \mathrm{PN}_{\hat{\mathrm{K}}}\right\}$, where $6.5 \mathrm{PN}_{\hat{\mathrm{K}}}$ indicates the tidal-tail term entering at $6.5 \mathrm{PN}$ order (i.e., neglecting the spin-tidal part entering at the same order). The asymptotic condition in Eq. (25) is satisfied by requiring only that including higher-order PN terms increases the agreement between the series and the full IMRPhenomD-NRTidal model, i.e., that $\Delta(x)$ decreases as more terms are added to the series. From Fig. 10 we notice that for frequencies approximately below $100 \mathrm{~Hz}$ this is indeed the case. However, this is not true anymore above $f \sim 100 \mathrm{~Hz}$ which roughly corresponds to the crossing between the $6 \mathrm{PN}$ order curve and the $6.5 \mathrm{PN}_{\hat{\mathrm{K}}}$ order curve in Fig. 10. In other words, including the $6.5 \mathrm{PN}_{\hat{\mathrm{K}}}$ order tidal-tail term makes the difference between the IMRPhenomD-NRTidal model and the PN series larger than that obtained retaining only terms up to $6 \mathrm{PN}$ order.

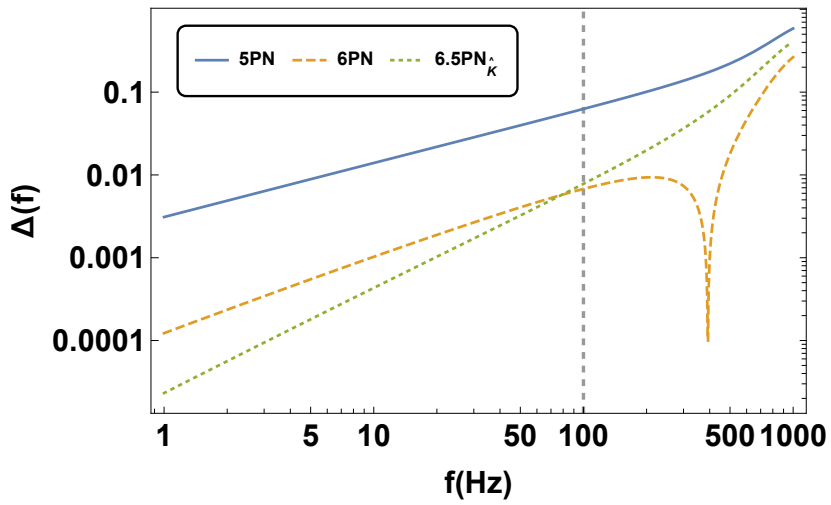

FIG. 10. Comparison between the TaylorF2 and PhenomD approximants following the criterion defined in Eq. (24). We show $\Delta(f)$ [cf. Eq. (25)] as a function of the frequency for the same set of tidal corrections. The vertical dashed line at $f=100 \mathrm{~Hz}$ fixes the approximate frequency where the discrepancies $\Delta(f)$ for the 6PN order and the $6.5 \mathrm{PN}_{\hat{\mathrm{K}}}$ order tidal-tail term cross each other for an equal-mass $2.8 M_{\odot}$ BNS system.

Based on this, we can assert that the addition of the $6.5 \mathrm{PN}_{\hat{\mathrm{K}}}$ order tail-tidal term will only improve the PN approximants in the low frequency regime. On the other hand, at frequencies $f \gtrsim 100 \mathrm{~Hz}$, the inclusion of this term decreases the agreement between the TaylorF2 approximant and the IMRPhenomDNRTidal model. Considering that current ground-based detectors collect most of the SNR around these frequencies, the inclusion of such a term would be magnified, producing a negative impact on parameter-estimation analyses.

It is also worth noting that, since the IMRPhenomDNRTidal model does not account for the spin-tidal interactions, we could not extend this analysis to the new $6.5 \mathrm{PN}_{\hat{\Lambda}}$ order spin-tidal contributions $\hat{\Lambda}$. This has prevented us from determining whether there exists a maximum frequency $x_{\max }$ for the $\hat{\Lambda}$ coefficient for which Eq. (24) is unfulfilled, thus not ensuring the correctness of such terms up to $f_{\max }=2048$. However, as it is shown in Fig. 2 and in agreement with the results of Sec. V, the order of magnitude of these terms is expected to be a factor $\sim 8$ smaller than the tidal-tail term for moderately high spins $\chi=0.1$. Therefore, the corrections being so small suggest that there are no such divergences for the $6.5 \mathrm{PN}_{\hat{\Lambda}}$ order term. For this reason, we set $f_{\text {max }}=2048$.

We quantify the impact of different PN tidal terms by applying the analysis described in Sec. III. In this case, we use as the reference model the full IMRPhenomD-NRTidal model matched against the tidal part of the TaylorF2 approximant truncated to $\left\{5 \mathrm{PN}, 6 \mathrm{PN}, 6.5 \mathrm{PN}_{\hat{\mathrm{K}}}, 6.5 \mathrm{PN}_{\hat{\Lambda}}\right\}$ order, respectively, in the frequency range $f \in(23,2048) \mathrm{Hz}$, consistent with the range used in [8]. The $6.5 \mathrm{PN}_{\hat{\mathrm{K}}}$ and $6.5 \mathrm{PN}_{\hat{\Lambda}}$ terms refer to two separate 6.5PN order models, where in the former we include only the tidal-tail term $\hat{K}$ while in the latter we set $\hat{K}=0$ but accounting for the spin-tail coefficient $\hat{\Lambda}$ with $\chi=0.2$.

In Fig. 11 we show the effect of adding sequentially new PN terms to the TaylorF2 approximant. The first term considered in this analysis is the leading-order 5PN term, which provides an estimated value of the weighted tidal deformability $\tilde{\Lambda}$ remarkably shifted about $130 \%$ from the injected one (blue curve). The offset is significantly reduced by adding the 6PN order term (orange curve) to the TaylorF2 approximant, which now makes the reconstructed $\tilde{\Lambda}$ a value $30 \%$ closer to the true one. This suggests that the $6 \mathrm{PN}$ order term still accounts for 


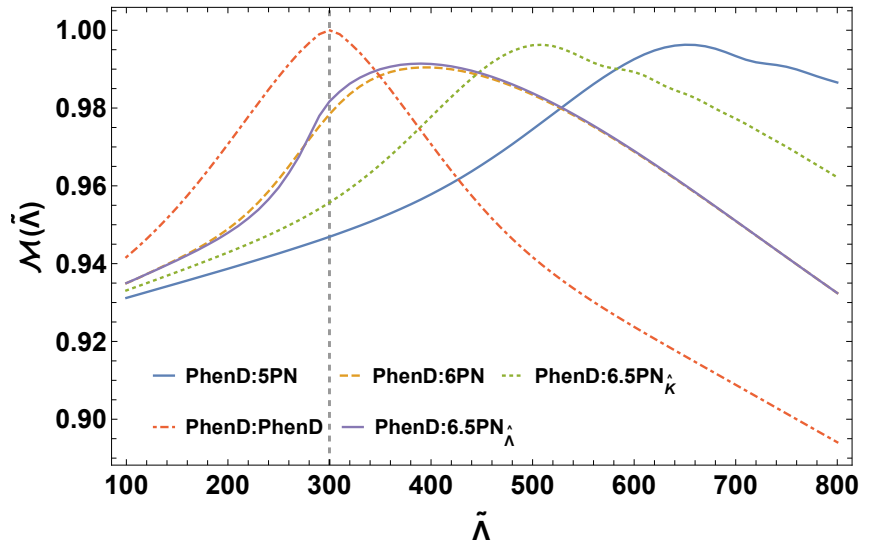

FIG. 11. Match as in Eq. (13) obtained by comparing the PhenomD model and the TaylorF2 approximant truncated at $\left\{5 \mathrm{PN}, 6 \mathrm{PN}, 6.5 \mathrm{PN}_{\hat{\mathrm{K}}}, 6.5 \mathrm{PN}_{\hat{\Lambda}}\right\}$ order. The $6.5 \mathrm{PN}_{\hat{\mathrm{K}}}$ and $6.5 \mathrm{PN}_{\hat{\Lambda}}$ terms refer to the tidal-tail and spin-tail coefficients respectively with $\chi=0.2$. The dashed vertical line determines the injected tidal deformability for an equal-mass binary with total mass $M=2.8 M_{\odot}$.

a non-negligible contribution to the tidal phase; thus it cannot be omitted for parameter estimation. Indeed, the TaylorF2 approximant up to 6PN order has been used to provide the first estimates to the leading-order tidal deformability in [1]. Interestingly, our analysis suggests that TaylorF2 waveforms always provide upper bounds on $\tilde{\Lambda}$ larger than those provided by NR-calibrated waveforms [1]. Furthermore, the addition of the next-to-next-to-leading-order $6.5 \mathrm{PN}_{\hat{\Lambda}}$ spin-tidal term (purple line) with $\chi=0.2$ just produces a minimal deviation with respect to the 6PN next-to-leading-order term due to the smallness of the spin-tidal interaction, which is in agreement with the results of Sec. V. On the contrary, the tail-tidal term (green curve) does not improve the agreement between the two waveform models but actually increases the systematic error. We believe that this is due to the fact that we are using such term in a regime where the PN series is poorly convergent, so that such term is no longer a good approximation to the true GW phase. Including (currently unknown ${ }^{10}$ ) higherorder PN terms could in principle correct this pathological behavior.

\section{CONCLUSION}

We have estimated the impact of the 6PN order magnetic TLNs terms described in [44-46] and the new 6.5PN order spin-tidal corrections computed by [46] on GW BNS events with physical parameters consistent with GW170807. We considered two different scenarios: a standard scenario in which we choose the physical parameters to be consistent with the median estimates provided by the LIGO-Virgo Collaboration [1, 8] and [7], and a more optimistic scenario based on a hypothetical optimally oriented GW170807-like event (thus increasing the SNR to $\rho=1750$ or to that compatible with the optimal observation of GW170817 under ET) and where the leading-order tidal deformability is taken to be $\tilde{\Lambda}=800$,

${ }^{10}$ Here we are not considering the EOB tidal model, in which some terms have been derived up to $7.5 \mathrm{PN}$ order [41]. i.e., consistent with the LIGO-Virgo upper limits. Given the high SNR produced in the two scenarios, we have taken advantage of the FIM formalism to compute the parameter bias induced by neglecting the magnetic TLNs and the spin-tidal terms on our waveform approximants. We provide estimates for both scenarios and for the eaLIGO and ET-D noise sensitivity curves.

We found that the internal dynamics induced on the NS fluid and encoded in the magnetic TLNs could be observed with third-generation GW detectors such as ET-D for our optimistic scenario. The effects of the magnetic TLNs, for both static and irrotational fluids, do not affect by more than $\sim 5 \%$ the estimate of $\tilde{\Lambda}$ when they are not included in current waveform template banks. This is explained by the fact that the magnetic TLNs are roughly a factor of 100 smaller than the corresponding electric TLNs, while they enter at the next-toleading PN order relative to the principal $\tilde{\Lambda}$. This is consistent with [28].

We find a slightly more optimistic scenario regarding the recently computed 6.5PN order spin-tidal couplings. In this case we have quantified for the first time the impact of these terms by means of the bias produced on the measurement of the tidal deformability $\tilde{\Lambda}$ that arises from neglecting these terms in our waveform approximants. We find that for a GW170817like BNS event detected by the ET in the optimal orientation, spin-tidal effects can be negligible unless the spins are at least $\left|\chi_{1,2}\right| \sim 0.07$ for $\tilde{\Lambda} \sim 800$ (which is the LIGO-Virgo upper limit on $\tilde{\Lambda}$ ) and $\left|\chi_{1,2}\right| \sim 0.15$ for the more conservative value $\tilde{\Lambda} \sim 300$. Therefore, these effects could be relevant for BNS waveform approximants only in the unlikely case that spinning binaries with $\chi_{1,2} \sim 0.1$ merge in our local universe. However, considering the current accuracy of the NR codes, we do not expect that the minimal variations produced by spin-tidal couplings can be separated from the numerical noise [13]. We focused on the planned ET detector [75-77], but similar results are expected for other third-generation designs, such as Cosmic Explorer [85, 86]. In particular, since the minimum sensitivity of the latter is a factor of a few better than ET, we expect that the effect of spin-tidal coupling should be slightly easier to detect.

Finally, we have studied the convergence properties of the high-PN order tidal terms. This is relevant for any study willing to add higher than 6PN order terms to TaylorF2 approximants. We found that though these terms contribute to increase the accuracy at the very low frequencies $(f \ll 100 \mathrm{~Hz})$, they do not satisfy the convergence properties at $f \sim 100 \mathrm{~Hz}$. Given that, for BNS events, most of the SNR is collected at frequencies that surround this value, the inclusion of such higher-order terms in the waveform models may lead to large systematic errors and to a significant bias on the parameter estimation. This explains why TaylorF2 approximants restricted to 6PN order produce results more compatible with the NR-calibrated models than the extended 6.5PN order tidaltail model, since the 6PN order term satisfies the convergence properties, whereas this is not the case of the tidal-tail term. We could not extend this analysis to the 6.5PN order spin-tidal coefficients since there is no NR-calibrated approximant accounting for these terms. Alternatively, a different resummation of the PN terms as in [42] could correct the pathological behavior of the series.

Future work will focus on the inclusion of the RTLN terms computed in Ref. [46], although this will have to wait until 
the conceptual problem related to the inclusion of the RTLNs in a Lagrangian formulation is solved. Another extension of our work is related to the analysis of systems for which the spin-tidal effects are expected to be larger, for instance in GW searches for exotic compact objects based on tidal effects [8789]. There is no reason to expect that such objects should be slowly spinning (this is particularly true for supermassive objects in the LISA band, whose spin might grow through accretion or through subsequent mergers during the galaxy evolution). We expect that the inclusion of the spin-tidal couplings computed here will improve previous analysis [89].

\section{ACKNOWLEDGMENTS}

We thank Evan Hall for making the computations of the Einstein Telescope geometrical factors available and Ilya Mandel for useful clarifications about our computations. We acknowledge support from the Amaldi Research Center funded by the MIUR program "Dipartimento di Eccellenza" (CUP: B81I1800101). P.P. acknowledges financial support provided under the European Union's H2020 ERC, Starting Grant Agreement No. DarkGRA-757480, and the kind hospitality of the Universitat de les Illes Balears, where part of this work was done. The authors acknowledge networking support by the COST Action CA16104. This project has received funding from the European Union's Horizon 2020 research and innovation program under the Marie Sklodowska-Curie Grant Agreement No 690904.

\section{Appendix A: APPROXIMATED CONVERSION OF GW170817 TO ET}

A GW strain $h(t)$ detected by some particular GW observatory in its own coordinate frame has the following form,

$$
h_{D}(t, \vec{\gamma}, \iota, \theta, \phi, \psi, \zeta)=D_{A}^{i j}(\theta, \phi, \psi, \zeta) h_{i j}(t, \vec{\gamma}, \iota),
$$

where $D_{A}^{i j}$ is the so-called detector tensor of the detector $A$ and $h_{i j}$ accounts for the GW strain tensor. Then, Eq. (A1) is nothing other than the projection of the strain tensor $h_{i j}$ defined in the source coordinate frame (where $\vec{\gamma}$ are the physical parameters and $\iota$ the source inclination) to the detector frame according to its sky location $\{\theta, \phi\}$, polarization angle $\psi$, and the angle between the detector arms $\zeta$. In other words, it gives the amount of signal traveling in the direction perpendicular to the detector plane and with polarization angle $\psi$. For instance, an interferometer with a pair of arms forming an angle $\zeta$, the detector tensor $D_{A}^{i j}$ reads

$$
D_{A}^{i j}=\frac{1}{2}\left[\begin{array}{cc}
F_{+}(\theta, \phi, \psi) \sin ^{2} \zeta & -F_{\times}(\theta, \phi, \psi) \cos \zeta \sin \zeta \\
-F_{\times}(\theta, \phi, \psi) \cos \zeta \sin \zeta & -F_{+}(\theta, \phi, \psi) \sin ^{2} \zeta
\end{array}\right],
$$

where $F_{+, \times}(\theta, \phi, \psi, \zeta)$ are the so-called detector antenna patterns. In parallel, the strain tensor $h_{i j}$ of an elliptically polarized GW traveling perpendicular to the detector frame is

$$
h_{i j}=h_{0}(t, \vec{\gamma})\left[\begin{array}{cc}
\frac{1+\cos ^{2} \iota}{2} & i \cos \iota \\
i \cos \iota & -\frac{1+\cos ^{2} \iota}{2}
\end{array}\right],
$$

consistent with the $h_{+}$(diagonal) and $h_{\times}$(antidiagonal) polarizations and noting that $\iota=0$ represents the case of the optimally oriented (circularly polarized) source considered in this work. For an L-shaped detector such as the LIGO-Virgo observatories $\left(\zeta=90^{\circ}\right)$ it is easy to show that combining equations (A1), (A2) and (A3) we get the usual expression for $h_{D}$,

$$
h_{D}(t, \vec{\gamma}, \iota, \theta, \phi, \psi, \zeta)=\left(\frac{1+\cos ^{2} \iota}{2}\right) F_{+}(\theta, \phi, \psi) h_{+}(t, \vec{\gamma}, i),
$$

which simplifies to

$$
h_{D}(t, \vec{\gamma}, \iota, \theta, \phi, \psi, \zeta)=\left(\frac{1+\cos ^{2} \iota}{2}\right) h_{0}(t, \vec{\gamma}),
$$

for the particular case of $\theta=\phi=\psi=0$.

On the other hand, the SNR collected by a network of (uncorrelated) detectors is given by

$$
\rho=\sqrt{\sum_{D} \rho_{D}^{2}}
$$

where $\rho_{D}$ accounts for the SNR observed by a single detector as described by Eq. (12). In the particular case of GW170817, its position in the sky implied that almost all the SNR was collected by the Hanford and Livingstone observatories [1] also with similar magnitudes. In this scenario, Eq. (A6) can be approximated by

$$
\rho_{H L}=\sqrt{\rho_{H}^{2}+\rho_{L}^{2}} \approx \sqrt{2} \rho_{H} \approx\left\{\begin{array}{l}
\rho_{H L}^{s t}=32.4 \\
\rho_{H L}^{o p t}=32.4\left(\frac{2}{1+\cos ^{2}\left(30^{\circ}\right)}\right)
\end{array}\right.
$$

where $\rho_{H, L}$ are the individual Hanford and Livingstone SNR's and the first line consistent with our standard scenario $\rho_{H L}^{s t}$ while in the second one $\rho_{H L}^{o p t}$ reproduces the same LIGO-Virgo event if $\iota$ would have been optimal. Alternatively, current prospects concerning the design of the ET geometry anticipate the construction of a detector formed by joining three separate interferometers in a triangular shape, that is, with $\zeta=60^{\circ}$ $[77,79]$ and with the three responses described by $D_{1}^{i j}, D_{2}^{i j}$ and $D_{3}^{i j}$ as

$$
D_{1,2}^{i j}=\frac{1}{2}\left[\begin{array}{cc} 
\pm \frac{3}{4} & -\frac{\sqrt{3}}{4} \\
-\frac{\sqrt{3}}{4} & \mp \frac{3}{4}
\end{array}\right], \quad D_{3}^{i j}=\frac{1}{2}\left[\begin{array}{cc}
0 & \frac{\sqrt{3}}{2} \\
\frac{\sqrt{3}}{2} & 0
\end{array}\right],
$$

where we have also assumed $\theta=\phi=\psi=0$. Then, using Eqs. (A1), (A6), and (A8), the total SNR in ET will result from adding up the three individual contributions as,

$$
\rho_{E T_{\Delta}}=\sqrt{\sum_{A=1,3} 4 \mathcal{R} \int_{f_{\min }}^{f_{\max }} \frac{\left|D_{A}^{i j} h_{i j}\right|^{2}}{S_{n}^{E T}(f)} d f}=f(\iota) \rho_{E T}
$$

and

$$
f(\iota)=\frac{3}{2} \sqrt{1 / 8\left(1+6 \cos ^{2} \iota+\cos ^{4} \iota\right)},
$$

where $\rho_{E T}$ is the SNR computed for a single L-squared ET detector for an optimally oriented source and $f(\iota)$ is a geometrical factor equal to $3 / 2$ for $\iota=0$ [79]. Then, by combining Eqs. (A7) and (A9), we get

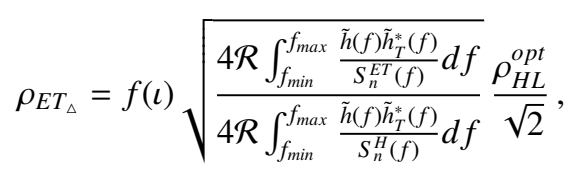


where $S_{n}^{E T, H}$ are the sensitivity curves taken from the literature for ET-D and eaLIGO, $f_{\text {min }}=\{3,30\}$ and $f_{\text {max }}=2048 \mathrm{~Hz}$ for both detectors, respectively. Finally, we get the following conversion factors:

$$
\rho_{E T_{\Delta}} \approx \rho_{H L}^{s t}\left\{\begin{array}{ll}
45 & \text { standard scenario } \\
55 & \text { optimal scenario }
\end{array} .\right.
$$

[1] B. Abbott et al. (Virgo, LIGO Scientific), Phys. Rev. Lett. 119, 161101 (2017), arXiv:1710.05832 [gr-qc].

[2] W. Del Pozzo, T. G. F. Li, M. Agathos, C. Van Den Broeck, and S. Vitale, Phys. Rev. Lett. 111, 071101 (2013), arXiv:1307.8338 [gr-qc].

[3] A. Bauswein, O. Just, H.-T. Janka, and N. Stergioulas, Astrophys. J. 850, L34 (2017), arXiv:1710.06843 [astro-ph.HE].

[4] E. R. Most, L. R. Weih, L. Rezzolla, and J. Schaffner-Bielich, (2018), arXiv:1803.00549 [gr-qc].

[5] I. Harry and T. Hinderer, (2018), arXiv:1801.09972 [gr-qc].

[6] B. P. Abbott et al. (Virgo, LIGO Scientific), (2018), arXiv:1805.11581 [gr-qc].

[7] S. De, D. Finstad, J. M. Lattimer, D. A. Brown, E. Berger, and C. M. Biwer, (2018), arXiv:1804.08583 [astro-ph.HE].

[8] B. P. Abbott et al. (Virgo, LIGO Scientific), (2018), arXiv: 1805.11579 [gr-qc].

[9] F. Özel and P. Freire, Ann. Rev. Astron. Astrophys. 54, 401 (2016), arXiv:1603.02698 [astro-ph.HE].

[10] K. G. Arun, A. Buonanno, G. Faye, and E. Ochsner, Phys. Rev. D79, 104023 (2009), [Erratum: Phys. Rev.D84,049901(2011)], arXiv:0810.5336 [gr-qc].

[11] A. Buonanno, B. Iyer, E. Ochsner, Y. Pan, and B. S. Sathyaprakash, Phys. Rev. D80, 084043 (2009), arXiv:0907.0700 [gr-qc].

[12] C. K. Mishra, A. Kela, K. G. Arun, and G. Faye, Phys. Rev. D93, 084054 (2016), arXiv:1601.05588 [gr-qc].

[13] T. Dietrich, S. Bernuzzi, and W. Tichy, Phys. Rev. D96, 121501 (2017), arXiv:1706.02969 [gr-qc].

[14] T. Dietrich and T. Hinderer, Phys. Rev. D95, 124006 (2017), arXiv:1702.02053 [gr-qc].

[15] T. Dietrich et al., (2018), arXiv:1804.02235 [gr-qc].

[16] R. Cotesta, A. Buonanno, A. Bohé, A. Taracchini, I. Hinder, and S. Ossokine, (2018), arXiv:1803.10701 [gr-qc].

[17] L. Santamaria et al., Phys. Rev. D82, 064016 (2010), arXiv:1005.3306 [gr-qc].

[18] M. Hannam, P. Schmidt, A. Bohé, L. Haegel, S. Husa, F. Ohme, G. Pratten, and M. Pürrer, Phys. Rev. Lett. 113, 151101 (2014), arXiv:1308.3271 [gr-qc].

[19] S. Husa, S. Khan, M. Hannam, M. Pürrer, F. Ohme, X. Jiménez Forteza, and A. Bohé, Phys. Rev. D93, 044006 (2016), arXiv:1508.07250 [gr-qc].

[20] S. Khan, S. Husa, M. Hannam, F. Ohme, M. Pürrer, X. Jiménez Forteza, and A. Bohé, Phys. Rev. D93, 044007 (2016), arXiv:1508.07253 [gr-qc].

[21] Y. Pan, A. Buonanno, A. Taracchini, L. E. Kidder, A. H. Mroué, H. P. Pfeiffer, M. A. Scheel, and B. Szilágyi, Phys. Rev. D89, 084006 (2014), arXiv:1307.6232 [gr-qc].

[22] M. Pürrer, Class. Quant. Grav. 31, 195010 (2014), arXiv:1402.4146 [gr-qc].

[23] M. Pürrer, Phys. Rev. D93, 064041 (2016), arXiv:1512.02248 [gr-qc].

[24] B. P. Abbott et al. (Virgo, LIGO Scientific), (2016), arXiv:1606.01210 [gr-qc].

[25] S. Babak, A. Taracchini, and A. Buonanno, Phys. Rev. D95, 024010 (2017), arXiv:1607.05661 [gr-qc].

[26] A. Bohé et al., Phys. Rev. D95, 044028 (2017), arXiv:1611.03703 [gr-qc].

[27] A. Buonanno and T. Damour, Phys. Rev. D59, 084006 (1999), arXiv:gr-qc/9811091 [gr-qc].
[28] S. Bernuzzi, A. Nagar, T. Dietrich, and T. Damour, Phys. Rev. Lett. 114, 161103 (2015), arXiv:1412.4553 [gr-qc].

[29] S. Bernuzzi, T. Dietrich, and A. Nagar, Phys. Rev. Lett. 115, 091101 (2015), arXiv:1504.01764 [gr-qc].

[30] T. Hinderer et al., Phys. Rev. Lett. 116, 181101 (2016), arXiv:1602.00599 [gr-qc].

[31] L. Blanchet, Living Rev. Rel. 9, 4 (2006).

[32] E. Poisson and C. M. Will, Gravity: Newtonian, PostNewtonian, Relativistic (Cambridge University Press, 2014).

[33] P. Pani, L. Gualtieri, and V. Ferrari, Phys. Rev. D92, 124003 (2015), arXiv:1509.02171 [gr-qc].

[34] P. Landry and E. Poisson, Phys. Rev. D91, 104026 (2015), arXiv:1504.06606 [gr-qc].

[35] P. Landry and E. Poisson, Phys. Rev. D91, 104018 (2015), arXiv:1503.07366 [gr-qc].

[36] P. Landry, Phys. Rev. D95, 124058 (2017), arXiv:1703.08168 [gr-qc].

[37] J. Gagnon-Bischoff, S. R. Green, P. Landry, and N. Ortiz, Phys. Rev. D97, 064042 (2018), arXiv:1711.05694 [gr-qc].

[38] T. Damour, B. R. Iyer, and B. S. Sathyaprakash, Phys. Rev. D63, 044023 (2001), [Erratum: Phys. Rev.D72,029902(2005)], arXiv:gr-qc/0010009 [gr-qc].

[39] T. Damour, B. R. Iyer, and B. S. Sathyaprakash, Phys. Rev. D66, 027502 (2002), arXiv:gr-qc/0207021 [gr-qc].

[40] K. G. Arun, B. R. Iyer, B. S. Sathyaprakash, and P. A. Sundararajan, Phys. Rev. D71, 084008 (2005), [Erratum: Phys. Rev.D72,069903(2005)], arXiv:gr-qc/0411146 [gr-qc].

[41] T. Damour, A. Nagar, and L. Villain, Phys. Rev. D85, 123007 (2012), arXiv:1203.4352 [gr-qc].

[42] A. Nagar et al., (2018), arXiv:1806.01772 [gr-qc].

[43] T. Binnington and E. Poisson, Phys. Rev. D80, 084018 (2009), arXiv:0906.1366 [gr-qc].

[44] K. Yagi, Phys. Rev. D89, 043011 (2014), [Erratum: Phys. Rev.D97,no.12,129901(2018)], arXiv:1311.0872 [gr-qc].

[45] B. Banihashemi and J. Vines, (2018), arXiv:1805.07266 [gr$\mathrm{qc}]$.

[46] T. Abdelsalhin, L. Gualtieri, and P. Pani, Phys. Rev. D98, 104046 (2018), arXiv:1805.01487 [gr-qc].

[47] P. Landry, (2018), arXiv:1805.01882 [gr-qc].

[48] E. E. Flanagan and T. Hinderer, Phys. Rev. D77, 021502 (2008), arXiv:0709.1915 [astro-ph].

[49] T. Hinderer, Astrophys. J. 677, 1216 (2008), arXiv:0711.2420 [astro-ph].

[50] T. Damour and A. Nagar, Phys. Rev. D80, 084035 (2009), arXiv:0906.0096 [gr-qc].

[51] E. Poisson, Phys. Rev. D91, 044004 (2015), arXiv:1411.4711 [gr-qc].

[52] P. Pani, L. Gualtieri, A. Maselli, and V. Ferrari, Phys. Rev. D92, 024010 (2015), arXiv:1503.07365 [gr-qc].

[53] E. Poisson and J. Doucot, Phys. Rev. D95, 044023 (2017), arXiv:1612.04255 [gr-qc].

[54] J. E. Vines and E. E. Flanagan, Phys. Rev. D88, 024046 (2013), arXiv:1009.4919 [gr-qc].

[55] J. Vines, E. E. Flanagan, and T. Hinderer, Phys. Rev. D83, 084051 (2011), arXiv:1101.1673 [gr-qc].

[56] R. P. Geroch, J. Math. Phys. 11, 2580 (1970).

[57] R. O. Hansen, J. Math. Phys. 15, 46 (1974).

[58] K. S. Thorne, Rev. Mod. Phys. 52, 299 (1980).

[59] V. Cardoso and L. Gualtieri, Class. Quant. Grav. 33, 174001 
(2016), arXiv:1607.03133 [gr-qc].

[60] J. Veitch et al., Phys. Rev. D91, 042003 (2015), arXiv:1409.7215 [gr-qc].

[61] K. Chatziioannou, A. Klein, N. Yunes, and N. Cornish, Phys. Rev. D95, 104004 (2017), arXiv:1703.03967 [gr-qc].

[62] C. Cutler and E. E. Flanagan, Phys. Rev. D49, 2658 (1994), arXiv:gr-qc/9402014 [gr-qc].

[63] M. Vallisneri, Phys. Rev. D77, 042001 (2008), arXiv:grqc/0703086 [GR-QC].

[64] L. Lindblom, B. J. Owen, and D. A. Brown, Phys. Rev. D78, 124020 (2008), arXiv:0809.3844 [gr-qc].

[65] M. Burgay, N. D’Amico, A. Possenti, R. N. Manchester, A. G. Lyne, B. C. Joshi, M. A. McLaughlin, M. Kramer, J. M. Sarkissian, F. Camilo, V. Kalogera, C. Kim, and D. R. Lorimer, Nature (London) 426, 531 (2003), astro-ph/0312071.

[66] D. A. Brown, I. Harry, A. Lundgren, and A. H. Nitz, Phys. Rev. D 86, 084017 (2012).

[67] W. Kastaun, F. Galeazzi, D. Alic, L. Rezzolla, and J. A. Font, Phys. Rev. D 88, 021501 (2013), arXiv:1301.7348 [gr-qc].

[68] A. Akmal, V. R. Pandharipande, and D. G. Ravenhall, Phys. Rev. C58, 1804 (1998), arXiv:nucl-th/9804027 [nucl-th].

[69] T. Dietrich, N. Moldenhauer, N. K. Johnson-McDaniel, S. Bernuzzi, C. M. Markakis, B. Bruegmann, and W. Tichy, (2015), arXiv:1507.07100 [gr-qc].

[70] P. Landry and E. Poisson, Phys. Rev. D92, 124041 (2015), arXiv:1510.09170 [gr-qc].

[71] P. Pani, L. Gualtieri, T. Abdelsalhin, and X. Jiménez-Forteza, Phys. Rev. D98, 124023 (2018), arXiv:1810.01094 [gr-qc].

[72] B. P. Abbott et al. (Virgo, LIGO Scientific), Astrophys. J. 832, L21 (2016), arXiv:1607.07456 [astro-ph.HE].

[73] B. S. Sathyaprakash and B. F. Schutz, Living Rev. Rel. 12, 2 (2009), arXiv:0903.0338 [gr-qc].

[74] J. Aasi et al. (VIRGO, LIGO Scientific), (2013), 10.1007/lrr2016-1, [Living Rev. Rel.19,1(2016)], arXiv:1304.0670 [gr-qc].

[75] S. Hild, S. Chelkowski, A. Freise, J. Franc, N. Morgado, R. Flaminio, and R. DeSalvo, Class. Quant. Grav. 27, 015003 (2010), arXiv:0906.2655 [gr-qc].
[76] B. Sathyaprakash et al., in Proceedings, 46th Rencontres de Moriond on Gravitational Waves and Experimental Gravity: La Thuile, Italy, March 20-27, 2011 (2011) pp. 127-136, arXiv:1108.1423 [gr-qc].

[77] B. Sathyaprakash et al., Gravitational waves. Numerical relativity - data analysis. Proceedings, 9th Edoardo Amaldi Conference, Amaldi 9, and meeting, NRDA 2011, Cardiff, UK, July 10-15, 2011, Class. Quant. Grav. 29, 124013 (2012), [Erratum: Class. Quant. Grav.30,079501(2013)], arXiv:1206.0331 [gr-qc].

[78] S. Hild et al., Class. Quant. Grav. 28, 094013 (2011), arXiv:1012.0908 [gr-qc].

[79] E. D. Hall, Personal communication at the $3 \mathrm{G}$ science team call (2018).

[80] C. Cutler et al., Phys. Rev. Lett. 70, 2984 (1993), arXiv:astro$\mathrm{ph} / 9208005$ [astro-ph].

[81] L. E. Simone, S. W. Leonard, E. Poisson, and C. M. Will, Class. Quant. Grav. 14, 237 (1997), arXiv:gr-qc/9610058 [gr-qc].

[82] N. Yunes and E. Berti, Phys. Rev. D77, 124006 (2008), [Erratum: Phys. Rev.D83,109901(2011)], arXiv:0803.1853 [gr-qc].

[83] Z. Zhang, N. Yunes, and E. Berti, Phys. Rev. D84, 024029 (2011), arXiv:1103.6041 [gr-qc].

[84] C. Bender and S. Orszag, Advanced Mathematical Methods for Scientists and Engineers I: Asymptotic Methods and Perturbation Theory (Springer New York, 2013).

[85] B. P. Abbott et al. (LIGO Scientific), Class. Quant. Grav. 34, 044001 (2017), arXiv:1607.08697 [astro-ph.IM].

[86] R. Essick, S. Vitale, and M. Evans, Phys. Rev. D96, 084004 (2017), arXiv:1708.06843 [gr-qc].

[87] V. Cardoso, E. Franzin, A. Maselli, P. Pani, and G. Raposo, Phys. Rev. D95, 084014 (2017), [Addendum: Phys. Rev.D95,no.8,089901(2017)], arXiv:1701.01116 [gr-qc].

[88] N. Sennett, T. Hinderer, J. Steinhoff, A. Buonanno, and S. Ossokine, Phys. Rev. D96, 024002 (2017), arXiv:1704.08651 [grqc].

[89] A. Maselli, P. Pani, V. Cardoso, T. Abdelsalhin, L. Gualtieri, and V. Ferrari, Phys. Rev. Lett. 120, 081101 (2018), arXiv:1703.10612 [gr-qc]. 